JOURNAL OF

SYMPLECTIC GEOMETRY

Volume 4, Number 2, 117-148, 2006

\title{
FIBERWISE VOLUME GROWTH VIA LAGRANGIAN INTERSECTIONS
}

\author{
Urs Frauenfelder and Felix Schlenk
}

We study certain topological entropy-type growth characteristics of Hamiltonian flows of a special form on the cotangent bundle $T^{*} M$ over a closed Riemannian manifold $(M, g)$. More precisely, the Hamiltonians generating the flows have to be of the form $H(t, q, p)=f(|p|)$ for $|p| \geq 1$, and the growth characteristics reflect how the Riemannian volumes of the unit balls in the fibers of $T^{*} M$ grow with the flow. Using a version of Lagrangian Floer homology and recent work of Abbondandolo and Schwarz, we obtain uniform lower bounds for the growth characteristics that depend only on $(M, g)$ or, more precisely, on the homology of the sublevel sets of the energy functional defined by the Riemannian metric $g$ on the space of based loops of Sobolev class $W^{1,2}$ in $M$. These lower bounds, in turn, can be estimated from below by purely topological characteristics of $M$. Our results in particular refine previous results of Dinaburg, Gromov, Paternain, and Paternain-Petean on the topological entropy of geodesic flows.

As an application, we obtain that for Riemannian manifolds, all of whose geodesics are closed (so-called $P$-manifolds), the fiberwise volume growth of every symplectomorphism in the symplectic isotopy class of the Dehn-Seidel twist is at least linear. This extends the main result of our paper $[\mathbf{3 0}]$ from the class of all currently known $P$-manifolds to all $P$-manifolds.

\section{Introduction and main results}

1.1. Topological entropy and volume growth. The topological entropy $h_{\text {top }}(\varphi)$ of a compactly supported $C^{1}$-diffeomorphism $\varphi$ of a smooth manifold $X$ is a basic numerical invariant measuring the orbit structure complexity of $\varphi$. There are various ways of defining $h_{\text {top }}(\varphi)$, see [39]. If $\varphi$ is $C^{\infty}$-smooth, a geometric way was found by Yomdin and Newhouse in their seminal works [76] and [54] respectively: Fix a Riemannian metric $g$ on $X$. For $j \in$ $\{1, \ldots, \operatorname{dim} X\}$ denote by $\Sigma_{j}$ the set of smooth compact (not necessarily 
closed) $j$-dimensional submanifolds of $X$ and by $\mu_{g}(\sigma)$ the volume of $\sigma \in \Sigma_{j}$ computed with respect to the measure on $\sigma$ induced by $g$. The $j$-th volume growth of $\varphi$ is defined as

$$
v_{j}(\varphi)=\sup _{\sigma \in \Sigma_{j}} \liminf _{m \rightarrow \infty} \frac{1}{m} \log \mu_{g}\left(\varphi^{m}(\sigma)\right),
$$

and the volume growth of $\varphi$ is defined as

$$
v(\varphi)=\max _{1 \leq j \leq \operatorname{dim} X} v_{j}(\varphi)
$$

Newhouse proved in [54] that $h_{\text {top }}(\varphi) \leq v(\varphi)$, and Yomdin proved in [76] that $h_{\text {top }}(\varphi) \geq v(\varphi)$ provided that $\varphi$ is $C^{\infty}$-smooth, so that

$$
h_{\text {top }}(\varphi)=v(\varphi) \text { if } \varphi \text { is } C^{\infty} \text {-smooth. }
$$

The topological entropy measures the exponential growth rate of the orbit complexity of a diffeomorphism. It therefore vanishes for many interesting dynamical systems. Following $[\mathbf{3 0}, \mathbf{4 0}]$, we thus also consider the $j$-th slow volume growth

$$
s_{j}(\varphi)=\sup _{\sigma \in \Sigma_{j}} \liminf _{m \rightarrow \infty} \frac{1}{\log m} \log \mu_{g}\left(\varphi^{m}(\sigma)\right)
$$

and the slow volume growth

$$
s(\varphi)=\max _{1 \leq j \leq \operatorname{dim} X} s_{j}(\varphi) .
$$

It measures the polynomial volume growth of the iterates of the most distorted smooth $j$-dimensional family of initial data. Note that $v_{j}(\varphi), v(\varphi)$, $s_{j}(\varphi), s(\varphi)$ do not depend on the choice of $g$ and that $v_{\operatorname{dim} X}(\varphi)=$ $s_{\operatorname{dim} X}(\varphi)=0$.

The aim of this paper is to give uniform lower estimates of localized versions of $v(\varphi)$ and $s(\varphi)$ for certain symplectomorphisms of cotangent bundles. We consider a smooth closed connected $d$-dimensional Riemannian manifold $(M, g)$ and the cotangent bundle $T^{*} M$ over $M$ endowed with the induced Riemannian metric $g^{*}$ and the standard symplectic form $\omega=\sum_{j=1}^{d} d p_{j} \wedge d q_{j}$. We abbreviate

$$
D(r)=\left\{(q, p) \in T^{*} M|| p \mid \leq r\right\} \quad \text { and } \quad D_{q}(r)=T_{q}^{*} M \cap D(r) .
$$

Let $\varphi$ be a $C^{1}$-smooth symplectomorphism of $\left(T^{*} M, \omega\right)$ which preserves $D(r)$. If $\varphi$ is $C^{\infty}$-smooth, (1.1) says that the maximal orbit complexity of $\left.\varphi\right|_{D(r)}$ is already contained in the orbit of a single submanifold of $D(r)$. Usually, lower estimates of the topological entropy do not give any information on the dimension or the location of such a submanifold. An attempt to localize such submanifolds for symplectomorphisms was made in [30], where 
we considered Lagrangian submanifolds only. In this paper, we further localize and consider for $\varphi$ as above the fiberwise volume growth

$$
\hat{v}_{\text {fibre }}(\varphi ; r)=\sup _{q \in M} \liminf _{m \rightarrow \infty} \frac{1}{m} \log \mu_{g^{*}}\left(\varphi^{m}\left(D_{q}(r)\right)\right)
$$

and the slow fiberwise volume growth

$$
\hat{s}_{\text {fibre }}(\varphi ; r)=\sup _{q \in M} \liminf _{m \rightarrow \infty} \frac{1}{\log m} \log \mu_{g^{*}}\left(\varphi^{m}\left(D_{q}(r)\right)\right) .
$$

In fact, we shall give uniform lower estimates of the (slow) volume growth of each fibre by considering the uniform fiberwise volume growth

$$
\check{v}_{\text {fibre }}(\varphi ; r)=\inf _{q \in M} \liminf _{m \rightarrow \infty} \frac{1}{m} \log \mu_{g^{*}}\left(\varphi^{m}\left(D_{q}(r)\right)\right)
$$

and the uniform slow fiberwise volume growth

$$
\check{s}_{\text {fibre }}(\varphi ; r)=\inf _{q \in M} \liminf _{m \rightarrow \infty} \frac{1}{\log m} \log \mu_{g^{*}}\left(\varphi^{m}\left(D_{q}(r)\right)\right) .
$$

Writing $v(\varphi ; r)=v\left(\left.\varphi\right|_{D(r)}\right)$ and so on, we clearly have

$$
\begin{aligned}
& v(\varphi ; r) \geq v_{d}(\varphi ; r) \geq \hat{v}_{\text {fibre }}(\varphi ; r) \geq \check{v}_{\text {fibre }}(\varphi ; r), \\
& s(\varphi ; r) \geq s_{d}(\varphi ; r) \geq \hat{s}_{\text {fibre }}(\varphi ; r) \geq \check{s}_{\text {fibre }}(\varphi ; r) .
\end{aligned}
$$

We shall obtain lower estimates of $\check{v}_{\text {fibre }}(\varphi ; r)$ and $\check{s}_{\text {fibre }}(\varphi ; r)$ in terms of the growth of certain homotopy-type invariants of $M$. We introduce these concepts of growth right now.

1.2. Energy hyperbolic manifolds. Let $(M, g)$ be a closed Riemannian manifold. We assume throughout that $M$ is connected. We fix $q_{0} \in M$ and denote by $\Omega^{1}\left(M, q_{0}\right)$ the space of all paths $q:[0,1] \rightarrow M$ of Sobolev class $W^{1,2}$ such that $q(0)=q(1)=q_{0}$. This space has a canonical Hilbert manifold structure [44]. The energy functional $\mathcal{E}=\mathcal{E}_{g}: \Omega^{1}\left(M, q_{0}\right) \rightarrow \mathbb{R}$ is defined as

$$
\mathcal{E}(q):=\frac{1}{2} \int_{0}^{1}|\dot{q}(t)|^{2} d t,
$$

where $|\dot{q}(t)|^{2}=g_{q(t)}(\dot{q}(t), \dot{q}(t))$. For $a>0$, we consider the sublevel sets

$$
\mathcal{E}^{a}\left(q_{0}\right):=\left\{q \in \Omega^{1}\left(M, q_{0}\right) \mid \mathcal{E}(q) \leq a\right\} .
$$

Let $\mathbb{P}$ be the set of non-negative integers which are prime or 0 , and set $\mathbb{F}_{p}=\mathbb{Z}_{p}$ and $\mathbb{F}_{0}=\mathbb{Q}$. Throughout, $\mathrm{H}_{*}$ denotes singular homology. Let

$$
\iota_{k}: \mathrm{H}_{k}\left(\mathcal{E}^{a}\left(q_{0}\right) ; \mathbb{F}_{p}\right) \longrightarrow \mathrm{H}_{k}\left(\Omega^{1}\left(M, q_{0}\right) ; \mathbb{F}_{p}\right)
$$

be the homomorphism induced by the inclusion $\mathcal{E}^{a}\left(q_{0}\right) \hookrightarrow \Omega^{1}\left(M, q_{0}\right)$. It is well known that for each $a$, the homology groups $\mathrm{H}_{k}\left(\mathcal{E}^{a}\left(q_{0}\right) ; \mathbb{F}_{p}\right)$ vanish for all large enough $k$, cf. [5], and so the sums in the following definition are finite. 
Definition 1.1. The Riemannian manifold $(M, g)$ is energy hyperbolic with exponent $C(M, g)$ if

$$
C(M, g):=\sup _{p \in \mathbb{P}} \liminf _{m \rightarrow \infty} \frac{1}{m} \log \sum_{k \geq 0} \operatorname{dim} \iota_{k} \mathrm{H}_{k}\left(\mathcal{E}^{(1 / 2) m^{2}}\left(q_{0}\right) ; \mathbb{F}_{p}\right)>0 .
$$

We also set

$$
c(M):=\sup _{p \in \mathbb{P}} \liminf _{m \rightarrow \infty} \frac{1}{\log m} \log \sum_{k \geq 0} \operatorname{dim} \iota_{k} \mathrm{H}_{k}\left(\mathcal{E}^{(1 / 2) m^{2}}\left(q_{0}\right) ; \mathbb{F}_{p}\right) .
$$

\section{Remarks.}

(a) The numbers $C(M, g)$ and $c(M)$ are less mysterious than they may look at first sight: They just measure the maximal exponential resp. polynomial homological growth of the energy sublevels $\mathcal{E}^{(1 / 2) m^{2}}\left(q_{0}\right)$ viewed as subsets of the full loop space $\Omega^{1}\left(M, q_{0}\right)$.

(b) Since $M$ is connected, $C(M, g)$ and $c(M)$ do not depend on $q_{0}$.

(c) Since $M$ is closed, neither the property "energy hyperbolic" nor $c(M)$ depend on $g$. We say that the closed manifold $M$ is energy hyperbolic if $(M, g)$ is energy hyperbolic for some and hence any Riemannian metric $g$ on $M$.

(d) For all $\lambda>0$, we have $C(M, \lambda g)=\frac{1}{\sqrt{\lambda}} C(M, g)$.

Examples of energy hyperbolic and non-energy hyperbolic Riemannian manifolds are given in Sections 1.4, 1.5, and 1.6.

1.3. Main result. Consider again a closed Riemannian manifold $(M, g)$, and let $H:[0,1] \times T^{*} M \rightarrow \mathbb{R}$ be a $C^{2}$-smooth Hamiltonian function meeting the following assumption: There exists $r_{H}>0$ and a function $f:[0, \infty) \rightarrow \mathbb{R}$ with $f^{\prime}\left(r_{H}\right) \neq 0$ such that

$$
H(t, q, p)=f(|p|) \quad \text { for }|p| \geq r_{H} .
$$

The Hamiltonian flow $\varphi_{H}^{t}$ of the time-dependent vector field $X_{H}$ given by $\omega\left(X_{H_{t}}, \cdot\right)=-d H_{t}(\cdot)$ is defined for all $t \in[0,1]$. We abbreviate $\varphi_{H}=\varphi_{H}^{1}$.

Theorem 1.2. Consider a closed Riemannian manifold $(M, g)$, and let $H:[0,1] \times T^{*} M \rightarrow \mathbb{R}$ be a $C^{2}$-smooth Hamiltonian function satisfying (1.4).

(i) If $(M, g)$ is energy hyperbolic, then

$$
\check{v}_{\text {fibre }}\left(\varphi_{H} ; r_{H}\right) \geq 2\left|f^{\prime}\left(r_{H}\right)\right| r_{H} C(M, g)>0 .
$$

(ii) In general,

$$
\check{s}_{\text {fibre }}\left(\varphi_{H} ; r_{H}\right) \geq c(M) \geq 1 .
$$

\section{Remarks.}

(a) As the identity mapping illustrates, the assumption $f^{\prime}\left(r_{H}\right) \neq 0$ in (1.4) is essential. 
(b) Theorem 1.2 extends well-known results from the study of geodesic flows, see [56, Corollary 3.9 and Chapter 5]: These results imply Theorem 1.2 if there exists an $\epsilon>0$ such that $H=\frac{1}{2}|p|^{2}$ on $D\left(r_{H}\right) \backslash D\left(r_{H}-\epsilon\right)$. In this situation, these results as well as Theorem 1.2 itself imply (i) with $\breve{v}_{\text {fibre }}\left(\varphi_{H} ; r_{H}\right)$ replaced by the uniform spherical volume growth

$$
\check{v}_{\text {sphere }}\left(\varphi_{H} ; r_{H}\right)=\inf _{q \in M} \liminf _{m \rightarrow \infty} \frac{1}{m} \log \mu_{g^{*}}\left(\varphi_{H}^{m}\left(\partial D_{q}\left(r_{H}\right)\right)\right) .
$$

(c) Assume that $M$ is energy hyperbolic, and let $\mathcal{G}(M)$ be the set of Riemannian metrics $g$ on $M$ with $\operatorname{Vol}(M, g)=1$. In view of Theorem $1.2(\mathrm{i})$, one may ask whether

$$
\inf \{C(M, g) \mid g \in \mathcal{G}(M)\}>0 .
$$

This is not so in general. Denote by $\varphi_{g}$ the geodesic flow on the unit sphere bundle $\partial D(1)$. Then,

$$
h_{\text {top }}(g):=h_{\text {top }}\left(\varphi_{g}\right)=v\left(\varphi_{g}\right) \geq \check{v}_{\text {sphere }}\left(\varphi_{g} ; 1\right) \geq 2 C(M, g)>0 .
$$

As shown in [58], many energy hyperbolic manifolds $M$ carry a sequence of Riemannian metrics $\left\{g_{n}\right\} \subset \mathcal{G}(M)$ such that $h_{\text {top }}\left(g_{n}\right) \rightarrow 0$ and thus $C\left(M, g_{n}\right) \rightarrow 0$.

In the following three sections, we describe lower bounds for $C(M, g)$ and $c(M)$ by homotopy-theoretical invariants of $M$. This will provide more computable lower bounds for fibre growth, will show that "most" closed manifolds are energy hyperbolic, and will reveal that Theorem 1.2 refines results of Dinaburg, Gromov, Paternain, and Paternain-Petean on the topological entropy of geodesic flows.

In general, our lower bounds for $C(M, g)$ and $c(M)$ will be the sum of two growths, namely the homological growth of (a part of) the based loop space $\Omega(\widetilde{M})$ of the universal cover of $M$ and the growth of the fundamental group $\pi_{1}(M)$. A direct approach combining both of these growths is given in Section 1.6. For pedagogical reasons, we first look at the case of finite $\pi_{1}(M)$, where the lower bounds come from $\Omega(\widetilde{M})$ alone, and then describe quantitative lower bounds provided by $\pi_{1}(M)$ alone.

1.4. Finite fundamental group. Consider a closed manifold $M$ with finite fundamental group $\pi_{1}(M)$. Let $\Omega\left(M, q_{0}\right)$ be the space of continuous paths $q:[0,1] \rightarrow M$ with $q(0)=q(1)=q_{0}$ endowed with the compact open topology. Since $M$ is connected, the homotopy type of $\Omega\left(M, q_{0}\right)$ does not depend on $q_{0}$ and is denoted $\Omega(M)$. For each $m \in \mathbb{N}$ and $p \in \mathbb{P}$, set

$$
r_{m}\left(M ; \mathbb{F}_{p}\right):=\sum_{k=0}^{m} \operatorname{dim} \mathrm{H}_{k}\left(\Omega(M) ; \mathbb{F}_{p}\right) .
$$


The manifold $M$ is called hyperbolic if for some $p \in \mathbb{P}$, the sequence $r_{m}\left(M ; \mathbb{F}_{p}\right)$ grows exponentially in $m$ (i.e., there exists $C>1$ such that $r_{m}\left(M ; \mathbb{F}_{p}\right) \geq C^{m}$ for all large enough $\left.m\right)$, and $M$ is called elliptic if for each $p \in \mathbb{P}$, the sequence $r_{m}\left(M ; \mathbb{F}_{p}\right)$ grows at most polynomially in $m$ (i.e., for each $p \in \mathbb{P}$, there exists $c_{p}<\infty$ such that $r_{m}\left(M ; \mathbb{F}_{p}\right) \leq m^{c_{p}}$ for all $\left.m\right)$. We set

$$
\rho(M):=\sup _{p \in \mathbb{P}} \liminf _{m \rightarrow \infty} \frac{1}{\log m} \log r_{m}\left(M ; \mathbb{F}_{p}\right) .
$$

If $M$ is elliptic, the supremum over $p \in \mathbb{P}$ is attained and $\rho(M)$ is finite [50]. It is conjectured that every closed manifold $M$ with finite $\pi_{1}(M)$ is either elliptic or hyperbolic. This is known if $\operatorname{dim} M \leq 5$, see [57, Section 3]. Moreover, the dichotomy " $r_{m}\left(M ; \mathbb{F}_{p}\right)$ grows either exponentially or polynomially" is known for $p=0$ and $p>\operatorname{dim} M$, and if $r_{m}\left(M ; \mathbb{F}_{p}\right)$ grows faster than polynomially, it grows at least like $C^{\sqrt{m}}$ for some $C>1$, see [19].

\section{Example 1.3.}

(a) It is easy to see that $M$ is elliptic resp. hyperbolic if and only if its universal cover $\widetilde{M}$ is, and that $\rho(M)=\rho(\widetilde{M})$, see Lemma 2.12(a). We thus assume that $M$ is simply connected. In dimensions 2 and 3, the standard sphere is the only such manifold up to diffeomorphism in view of the proof of the Poincaré conjecture; it is elliptic. In higher dimensions, "most" simply connected manifolds are hyperbolic. In dimension 4 , the simply connected elliptic manifolds up to homeomorphism are

$$
S^{4}, \quad \mathbb{C P}^{2}, \quad S^{2} \times S^{2}, \quad \mathbb{C P}^{2} \# \overline{\mathbb{C P}}^{2}, \quad \mathbb{C P}^{2} \# \mathbb{C P}^{2},
$$

and in dimension 5, the simply connected elliptic manifolds up to diffeomorphism are

$$
S^{5}, \quad S^{2} \times S^{3}, \quad S^{2} \ltimes S^{3}, \quad \mathrm{SU}(3) / \mathrm{SO}(3),
$$

where $S^{2} \ltimes S^{3}$ is the non-trivial $S^{3}$-bundle over $S^{2}$, see [57]. If $M$ is a sphere or $\mathbb{C P}^{2}$, then $\rho(M)=1$ (see Section 1.7), and for the six other manifolds above, $\rho(M)=2$. For the above 5-manifolds, a proof is given in [57, Section 3], and a variation of their proof also applies to the above 4-manifolds.

(b) Examples with $\rho(M)<2$ are rare in view of McCleary's theorem from [49]. If $\rho(M)<2$, then $\rho(M)=1$. Equivalently, the (reduced) integral cohomology ring of the universal cover of $M$ is generated by one element and hence agrees with the cohomology ring of a compact rank one symmetric space (CROSS). This implies further restrictions on the topology of $M$, see Section 3.

(c) Fix a prime number $p \in \mathbb{P}$, and let $M_{p}$ be a simply connected 5-manifold with $\mathrm{H}_{2}\left(M_{p} ; \mathbb{Z}\right)=\mathbb{F}_{p} \oplus \mathbb{F}_{p}$, see $[\mathbf{6 8}]$. Then the sequence 
$r_{m}\left(M_{p} ; \mathbb{F}_{p}\right)$ grows exponentially, while for all $p^{\prime} \in \mathbb{P} \backslash\{p\}$, the sequence $r_{m}\left(M_{p} ; \mathbb{F}_{p^{\prime}}\right)$ grows linearly, see [57, Section 3].

We refer to $[\mathbf{1 8 - 2 0 , 4 5 , 4 6 , 5 7 ]}$ and the references therein for more information on elliptic and hyperbolic manifolds. Using results of Serre, Gromov, and Benci, we shall prove

Proposition 1.4. Consider a closed manifold $M$ with $\pi_{1}(M)$ finite.

(i) $M$ is hyperbolic if and only if $M$ is energy hyperbolic.

(ii) In general, $c(M)=\rho(M) \geq 1$.

Suppose that the conjectured dichotomy " $M$ is either elliptic or hyperbolic" is true. Proposition 1.4 then implies that a closed manifold $M$ with finite $\pi_{1}(M)$ is either energy hyperbolic or elliptic with $c(M)$ finite.

Discussion 1.5. Theorem 1.2 and Proposition 1.4 show that for a closed Riemannian manifold $(M, g)$ with finite $\pi_{1}(M)$ and $H$ as in Theorem 1.2,

(i) $\check{v}_{\text {fibre }}\left(\varphi_{H} ; r_{H}\right)>0$ if $M$ is hyperbolic,

(ii) $\check{s}_{\text {fibre }}\left(\varphi_{H} ; r_{H}\right) \geq c(M)=\rho(M)=1$ in general.

ad (i). If $H$ is $C^{\infty}$-smooth, then $h_{\text {top }}\left(\varphi_{H} ; r_{H}\right) \geq \check{v}_{\text {fibre }}\left(\varphi_{H} ; r_{H}\right)$ by Yomdin's theorem and (1.2). Together with (i) we find that the topological entropy $h_{\text {top }}\left(\varphi_{H} ; r_{H}\right)$ is positive. This implies and is implied by the positivity of the topological entropy $h_{\text {top }}(g)$ of the geodesic flow of a $C^{\infty}$-smooth Riemannian metric $g$ on a hyperbolic manifold, a result found by Gromov and Paternain [56, Corollary 5.21].

ad (ii). There exist Riemannian manifolds and Hamiltonians $H$ with $\check{s}_{\text {fibre }}\left(\varphi_{H} ; r\right)=1$ for all large enough $r$, see Discussion 1.15 (a). The estimate in (ii) is thus sharp.

1.5. Infinite fundamental group. Assume now that $\pi_{1}(M)$ is infinite. Since $M$ is closed, $\pi_{1}(M)$ is then an infinite finitely presented group. Consider, more generally, an infinite finitely generated group $\Gamma$. The growth function $\gamma_{S}$ associated with a finite set $S$ of generators of $\Gamma$ is defined as follows: For each positive integer $m$, let $\gamma_{S}(m)$ be the number of distinct group elements which can be written as words with at most $m$ letters from $S \cup S^{-1}$. As is easy to see, [53], the limit

$$
\nu(S):=\lim _{m \rightarrow \infty} \frac{\log \gamma_{S}(m)}{m} \in[0, \infty)
$$

exists, and the property $\nu(S)>0$ is independent of the choice of $S$. In this case, the group $\Gamma$ is said to have exponential growth.

Example 1.6. If a closed manifold $M$ admits a Riemannian metric of negative sectional curvature, then $\pi_{1}(M)$ has exponential growth [53]. For $d=2$, the converse to this statement holds true, while for $d \geq 3$ there are closed $d$-dimensional manifolds for which $\pi_{1}(M)$ has exponential growth 
and which carry no Riemannian metric with negative sectional curvature, see [48, Corollaire III.10] and [16, p. 190].

If $\Gamma$ does not have exponential growth, $\Gamma$ is said to have subexponential growth. In this case, the degree of polynomial growth

$$
\sigma(\Gamma):=\liminf _{m \rightarrow \infty} \frac{\log \gamma_{S}(m)}{\log m} \in[0, \infty]
$$

is independent of the choice of $S$. The group $\Gamma$ has intermediate growth if $\sigma(\Gamma)=\infty$ and polynomial growth if $\sigma(\Gamma)<\infty$. While there are finitely generated groups of intermediate growth $[\mathbf{1 6}, \mathbf{3 2}]$, it is still unknown whether there are finitely presented groups of intermediate growth; we shall thus not consider a more refined notion of growth for such groups. According to a theorem of Gromov [34], the group $\Gamma$ has polynomial growth if and only if $\Gamma$ has a nilpotent subgroup $\Delta$ of finite index. Let $\left(\Delta_{k}\right)_{k \geq 1}$ be its lower central series defined inductively by $\Delta_{1}=\Delta$ and $\Delta_{k+1}=\left[\Delta, \Delta_{k}\right]$. Then,

$$
\sigma(\Gamma)=\sum_{k \geq 1} k \operatorname{dim}\left(\left(\Delta_{k} / \Delta_{k+1}\right) \otimes_{\mathbb{Z}} \mathbb{Q}\right),
$$

see $[\mathbf{4}, \mathbf{3 6}, \mathbf{6 9}, \mathbf{7 4}]$. We, in particular, see that $\sigma(\Gamma)$ is a positive integer.

\section{Example 1.7.}

(a) For the fundamental group of the torus, $\sigma\left(\mathbb{Z}^{d}\right)=1 \cdot d=d$.

(b) For the Heisenberg group

$$
\Gamma=\left\{\left(\begin{array}{lll}
1 & 0 & 0 \\
x & 1 & 0 \\
z & y & 1
\end{array}\right) \mid x, y, z \in \mathbb{Z}\right\},
$$

we have $\Delta_{1}=\Gamma$ and $\Delta_{2}=\{M(x, y, z) \in \Gamma \mid x=y=0\} \cong \mathbb{Z}$ and $\Delta_{k}=\{e\}$ for $k \geq 3$, so that $\sigma(\Gamma)=1 \cdot 2+2 \cdot 1=4$.

Much more information on growth of finitely generated groups can be found in $[\mathbf{1 6}]$.

Consider now a closed Riemannian manifold $(M, g)$ such that $\pi_{1}(M)$ has exponential growth. Given a finite set $S$ of generators, let $\ell(S, g)$ be the smallest real number such that for each $q \in M$ each generator $s \in S$ of $\pi_{1}(M) \cong \pi_{1}(M, q)$ can be represented by a smooth loop based at $q$ of length no more than $\ell(S, g)$. Then set

$$
\nu(M, g)=\sup \frac{\nu(S)}{\ell(S, g)}
$$

where the supremum is taken over all finite sets $S$ generating $\pi_{1}(M)$. A simple argument will prove

Proposition 1.8. Consider a closed Riemannian manifold $(M, g)$ with $\pi_{1}(M)$ infinite. 
(i) If $\pi_{1}(M)$ has exponential growth, $(M, g)$ is energy hyperbolic with $C(M, g) \geq \nu(M, g)$.

(ii) In general, $c(M) \geq \sigma\left(\pi_{1}(M)\right) \geq 1$.

In small dimensions, the converse to Proposition 1.8 (i) holds true. Assume that $\operatorname{dim} M \leq 3$, and if $\operatorname{dim} M=3$ also assume that the orientation cover of $M$ is geometrizable. ${ }^{1}$ Then, $\pi_{1}(M)$ has exponential growth if and only if $M$ is energy hyperbolic. We refer to $[\mathbf{2}, \mathbf{3 1}]$ for a proof.

Discussion 1.9. Theorem 1.2 and Proposition 1.8 show that for a closed Riemannian manifold $(M, g)$ with infinite $\pi_{1}(M)$ and $H$ as in Theorem 1.2,

(i) $\check{v}_{\text {fibre }}\left(\varphi_{H} ; r_{H}\right) \geq 2\left|f^{\prime}\left(r_{H}\right)\right| r_{H} \nu(M, g)$ if $\pi_{1}(M)$ has exponential growth,

(ii) $\check{s}_{\text {fibre }}\left(\varphi_{H} ; r_{H}\right) \geq c(M) \geq \sigma\left(\pi_{1}(M)\right) \geq 1$ in general.

ad (i). Yomdin's theorem and (i) yield the positive lower bound

$$
h_{\text {top }}(g) \geq 2 \nu(M, g)
$$

for the topological entropy of the geodesic flow of a $C^{\infty}$-smooth Riemannian metric $g$ on a manifold whose fundamental group has exponential growth. This is a version of Dinaburg's theorem, which holds for $C^{2}$-smooth $g$, (see [15] or [56, Theorem 5.18]).

ad (ii). For the flat torus $T^{d}$ and $H=\frac{1}{2} p^{2}$, we have $\check{s}_{\text {fibre }}\left(\varphi_{H} ; r\right)=$ $\sigma\left(\mathbb{Z}^{d}\right)=d$ for all $r>0$, so that (ii) is sharp.

1.6. Arbitrary fundamental group. In Sections 1.4 and 1.5, lower bounds for $C(M, g)$ and $c(M)$ have been obtained via the homological growth of $\Omega(M)$ if $\pi_{1}(M)$ is finite and via the growth of $\pi_{1}(M)$ if $\pi_{1}(M)$ is infinite. If $\pi_{1}(M)$ is infinite but of subexponential growth, the bound $c(M) \geq \sigma\left(\pi_{1}(M)\right)$ from Proposition 1.8 (ii) is often far from optimal. Indeed, recent work of Paternain and Petean [58] implies that in this situation, a better lower bound for $c(M)$ or even energy hyperbolicity of $M$ can often be established by adding to the growth of $\pi_{1}(M)$ the homological growth coming from the based loop space of a suitable simply connected complex in $M$.

Given a continuous map $f:(X, x) \rightarrow(Y, f(x))$ between path-connected pointed spaces, let $\Omega(f): \Omega(X) \rightarrow \Omega(Y)$ be the induced map between based loop spaces and let

$$
\mathrm{H}_{*}\left(\Omega(f) ; \mathbb{F}_{p}\right): \mathrm{H}_{*}\left(\Omega(X) ; \mathbb{F}_{p}\right) \longrightarrow \mathrm{H}_{*}\left(\Omega(Y) ; \mathbb{F}_{p}\right)
$$

be the map induced in homology. We say that a closed connected manifold $M$ is hyperbolic if there exists a finite simply connected CW complex $K$ and

\footnotetext{
${ }^{1}$ Thurston's geometrization conjecture says that any closed orientable 3-manifold is geometrizable. This conjecture has been proven in many cases, see [10, Section 4], and Perelman's work presumably implies it in general.
} 
a continuous map $f: K \rightarrow M$ such that for some $p \in \mathbb{P}$, the sequence

$$
r_{m}\left(M, K, f ; \mathbb{F}_{p}\right):=\sum_{k=0}^{m} \operatorname{rank} \mathrm{H}_{k}\left(\Omega(f) ; \mathbb{F}_{p}\right)
$$

grows exponentially in $m$. In general, we set

$$
\rho(M):=\sup _{(K, f)} \sup _{p \in \mathbb{P}} \liminf _{m \rightarrow \infty} \frac{1}{\log m} \log r_{m}\left(M, K, f ; \mathbb{F}_{p}\right),
$$

where the latter supremum is taken over all finite simply connected CW complexes $K$ and continuous maps $f: K \rightarrow M$. Notice that in the case of finite $\pi_{1}(M)$, the term "hyperbolic" and $\rho(M)$ were defined in Section 1.4 in a different way. An elementary argument shows that these definitions are equivalent, cf. Lemma 2.12 (a).

Example 1.10. Let $M$ be a closed 4- or 5-manifold with $\pi_{1}(M)$ infinite and of subexponential growth. As shown in $[\mathbf{3 8}, \mathbf{5 8}]$, "most" such manifolds are hyperbolic:

If $\operatorname{dim} M=4$, then $M$ is hyperbolic unless $M$ is finitely covered by $S^{3} \times S^{1}$ or by a manifold $s$-cobordant to $S^{2} \times T^{2}$ or unless $M$ is aspherical. In particular, $T^{4} \# \overline{\mathbb{C P}}^{2}$ is hyperbolic.

If $\operatorname{dim} M=5$, then $M$ is hyperbolic unless its universal cover has the rational homotopy type of a finite simply connected elliptic CW-complex.

The papers $[\mathbf{3 8}, \mathbf{5 8}]$ contain many further restrictions on the topology of non-hyperbolic manifolds with infinite $\pi_{1}(M)$.

Using an extension of Gromov's theorem from [33] proved in [58], we shall obtain

Proposition 1.11. Consider an arbitrary closed connected manifold $M$.

(i) If $M$ is hyperbolic, then $M$ is energy hyperbolic.

(ii) In general, $c(M) \geq \rho(M)+\sigma\left(\pi_{1}(M)\right) \geq 1$.

Discussion 1.12. Theorem 1.2 and Proposition 1.11 show that for a closed Riemannian manifold $(M, g)$ and for $H$ as in Theorem 1.2,

(i) $\check{v}_{\text {fibre }}\left(\varphi_{H} ; r_{H}\right)>0$ if $M$ is hyperbolic,

(ii) $\check{s}_{\text {fibre }}\left(\varphi_{H} ; r_{H}\right) \geq c(M) \geq \rho(M)+\sigma\left(\pi_{1}(M)\right) \geq 1$ in general.

ad (i). Again, Yomdin's theorem and (i) imply the positivity of the topological entropy of the geodesic flow of a $C^{\infty}$-smooth Riemannian metric $g$ on a hyperbolic manifold with $\pi_{1}(M)$ infinite, a result found by Paternain and Petean in [58].

ad (ii). Choose $m_{1}, \ldots, m_{l} \geq 2$ and $n \geq 1$, and endow $M=S^{m_{1}} \times \cdots \times$ $S^{m_{l}} \times T^{n}$ with the usual product Riemannian metric. For $H=\frac{1}{2}|p|^{2}$, we have $\check{s}_{\text {fibre }}\left(\varphi_{H} ; r\right)=l+n=\rho(M)+\sigma\left(\pi_{1}(M)\right)$ for all $r>0$, so that (ii) is sharp.

Given a Riemannian manifold $(M, g)$, we denote by $\varphi_{g}$ its geodesic flow on $T^{*} M$. The numbers $s(g):=s\left(\varphi_{g} ; r\right)$ and $\check{s}_{\text {fibre }}(g):=\check{s}_{\text {fibre }}\left(\varphi_{g} ; r\right)$ do not 
depend on $r>0$. Note that for $M=S^{1}$, we have $s(g)=\check{s}_{\text {fibre }}(g)=$ $\rho(M)+\sigma\left(\pi_{1}(M)\right)=1$.

Question 1.13. Consider a closed Riemannian manifold $(M, g)$ with infinite $\pi_{1}(M)$.

(a) Does $\rho(M)+\sigma\left(\pi_{1}(M)\right)<2$ imply $M=S^{1}$ ?

(b) Does $s(g)=1$ or $\check{s}_{\text {fibre }}(g)=1$ imply $M=S^{1}$ ?

A partial answer to Question 1.13 in the affirmative is given in [31].

1.7. Volume growth in the component of the Dehn-Seidel twist. A $P$-manifold is a connected Riemannian manifold all of whose geodesics are periodic. Such manifolds are closed and except for $S^{1}$ have finite fundamental group. As we shall see in Section 3, every $P$-manifold $M$ different from $S^{1}$ is elliptic with $c(M)=\rho(M)=1$. The known $P$-manifolds are the CROSSes

$$
S^{d}, \quad \mathbb{R P}^{d}, \quad \mathbb{C P}^{n}, \quad \mathbb{H} \mathrm{P}^{n}, \quad \mathbb{C}^{a} \mathrm{P}^{2}
$$

with their canonical Riemannian structures, their Riemannian quotients (which are all known), and so-called Zoll manifolds, which are modelled on spheres. It is an open problem whether there are other $P$-manifolds. More information on $P$-manifolds can be found in $[\mathbf{8}, \mathbf{3 0}]$, Section 10.10 of [7], and Section 3.

Let $(M, g)$ be a $P$-manifold. It is known that the unit-speed geodesics of $(M, g)$ admit a common period, and we shall assume $g$ to be scaled so that the minimal common period is 1 . We choose a smooth function $f:[0, \infty) \rightarrow$ $[0, \infty)$ such that

$$
f(r)=\frac{1}{2} r^{2} \quad \text { near } 0 \quad \text { and } \quad f^{\prime}(r)=1 \quad \text { for } r \geq 1,
$$

and following $[\mathbf{3}, \mathbf{6 5}, \mathbf{6 6}]$, we define the (left-handed) Dehn-Seidel twist $\vartheta_{f}$ to be the time-1-map of the Hamiltonian flow generated by $f(|p|)$. Since $(M, g)$ is a $P$-manifold, $\vartheta_{f}$ is the identity on $T^{*} M \backslash T_{1}^{*} M$, so that $\vartheta_{f}$ is a compactly supported symplectomorphism, $\vartheta_{f} \in \operatorname{Symp}^{c}\left(T^{*} M\right)$. We shall write $\vartheta$ for any map $\vartheta_{f}$ with $f$ satisfying (1.7). The class $[\vartheta]$ in the symplectic mapping class group $\pi_{0}\left(\operatorname{Symp}^{c}\left(T^{*} M\right)\right)$ clearly does not depend on $f$. Given $\varphi \in$ $\operatorname{Symp}^{c}\left(T^{*} M\right)$, we set $\check{s}_{\text {fibre }}(\varphi)=\check{s}_{\text {fibre }}(\varphi ; r)$, where $r>0$ is any number such that $\varphi$ is supported in $D(r)$. In Section 2.5, we shall use Theorem 1.2 (ii) to prove

Corollary 1.14. Let $(M, g)$ be a P-manifold, and let $\vartheta$ be a twist on $T^{*} M$. If $\varphi \in \operatorname{Symp}^{c}\left(T^{*} M\right)$ is such that $[\varphi]=\left[\vartheta^{m}\right] \in \pi_{0}\left(\operatorname{Symp}^{c}\left(T^{*} M\right)\right)$ for some $m \in \mathbb{Z} \backslash\{0\}$, then $\check{s}_{\text {fibre }}(\varphi) \geq 1$.

\section{Discussion 1.15.}

(a) A computation given in [30, Proposition $2.2(\mathrm{i})]$ shows that $s\left(\vartheta_{f}^{m}\right)=$ $\check{s}_{\text {fibre }}\left(\vartheta_{f}^{m}\right)=1$ for every $m \in \mathbb{Z} \backslash\{0\}$ and every $f$. Corollary 1.14 is 
thus sharp and shows that twists minimize slow volume growths in their symplectic isotopy class.

(b) Assume that $\varphi \in \operatorname{Symp}^{c}\left(T^{*} M\right) \backslash\{\mathrm{id}\}$ is such that $[\varphi]=\left[\vartheta^{0}\right]=[\mathrm{id}]$. If $d \geq 2$, this means that $\varphi$ is a non-identical compactly supported Hamiltonian diffeomorphism of $T^{*} M$. If the support of $\varphi$ misses some fibre, then $\check{s}_{\text {fibre }}(\varphi)=0$. On the other hand, combining a result in $[\mathbf{2 8}]$ with the arguments in $[\mathbf{6 0}]$ one finds that $s_{1}(\varphi) \geq 1$, see [29]. It is not hard to construct examples with $s(\varphi)=s_{j}(\varphi)=1$ for all $j \in\{1, \ldots, 2 d-1\}$, see $[29]$.

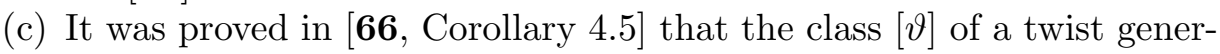
ates an infinite cyclic subgroup of $\pi_{0}\left(\operatorname{Symp}^{c}\left(T^{*} M\right)\right)$. Corollary 1.14 yields another proof of this.

(d) Corollary 1.14 was proved in [30] for all currently known $P$-manifolds. The proof there only used Lagrangian Floer homology and a symmetry argument for $\left(\mathbb{C P}^{n}, g_{\text {can }}\right)$.

We conclude with a question motivated by results in Section 3. For a closed Riemannian manifold $(M, g)$ with finite $\pi_{1}(M)$,

$$
s(g) \geq \check{s}_{\text {fibre }}(g) \geq c(M)=\rho(M) \geq 1
$$

according to (1.3) and Discussion 1.5 (ii). For a $P$-manifold, the computation in $[\mathbf{3 0}]$ shows that $s(g)=1$, so that all numbers in $(1.8)$ are 1 . Conversely, $\rho(M)=1$ implies that $\mathrm{H}^{*}(\widetilde{M} ; \mathbb{Z})$ is the cohomology ring of a CROSS and hence of a $P$-manifold in view of Mc Cleary's theorem mentioned in Example 1.3 (b).

Question 1.16. Consider a closed Riemannian manifold $(M, g)$ with finite $\pi_{1}(M)$.

(a) Does $\rho(M)=1$ imply that $M$ is homotopy equivalent to a $P$-manifold?

(b) Does $s(g)=1$ or $\check{s}_{\text {fibre }}(g)=1$ imply that $(M, g)$ is a P-manifold?

Question 1.16 (a) is partially answered and further discussed in Section 3.

\section{Proofs}

2.1. Proof of Theorem 1.2. Our proof is along the following lines. Using an idea from [30], we first show that fiberwise volume growth is a consequence of the growth of the dimension of certain Floer homology groups. Applying the isotopy invariance of Floer homology and a recent result of Abbondandolo and Schwarz, these homology groups are then seen to be isomorphic to the homology of the space of based loops in $M$ not exceeding a certain energy. 


\section{Step 1. Volume growth via Floer homology}

Geometric set-up. Consider a closed Riemannian manifold $(M, g)$ and a Hamiltonian $H:[0,1] \times T^{*} M \rightarrow \mathbb{R}$ as in Theorem 1.2. We abbreviate $\beta=$ $f^{\prime}\left(r_{H}\right)$, and we assume without loss of generality that $\beta>0$. Fix $\epsilon>0$ and let $K:[0,1] \times T^{*} M \rightarrow \mathbb{R}$ be a $C^{\infty}$-smooth function such that

$$
K(t, q, p)=\beta|p|^{2} \quad \text { if }|p| \geq r_{H}+\epsilon .
$$

For $m=1,2, \ldots$, we recursively define $K_{t}^{m}=K_{t}+K_{t}^{m-1} \circ\left(\varphi_{K}^{t}\right)^{-1}$. Then $\varphi_{K}^{m}=\varphi_{K}$. For $q_{0}, q_{1} \in M$, let $\Omega^{1}\left(T^{*} M, q_{0}, q_{1}\right)$ be the space of all paths $x:[0,1] \rightarrow T^{*} M$ of Sobolev class $W^{1,2}$ such that $x(0) \in T_{q_{0}}^{*} M$ and $x(1) \in T_{q_{1}}^{*} M$. This space has a canonical Hilbert manifold structure [44]. The action functional of classical mechanics $\mathcal{A}_{K^{m}}: \Omega^{1}\left(T^{*} M, q_{0}, q_{1}\right) \rightarrow \mathbb{R}$ associated with $K^{m}$ is defined as

$$
\mathcal{A}_{K^{m}}(x)=\int_{0}^{1}\left(\lambda(\dot{x})-K^{m}(t, x)\right) d t,
$$

where $\lambda=\sum_{j=1}^{d} p_{j} d q_{j}$ is the canonical 1-form on $T^{*} M$. This functional is $C^{\infty}$-smooth, and its critical points are precisely the elements of the space $\mathcal{P}\left(q_{0}, q_{1}, K^{m}\right)$ of $C^{\infty}$-smooth paths $x:[0,1] \rightarrow T^{*} M$ solving

$$
\dot{x}(t)=X_{K^{m}}(x(t)), \quad t \in[0,1], \quad x(j) \in T_{q_{j}}^{*} M, \quad j=0,1 .
$$

Notice that the elements of $\mathcal{P}\left(q_{0}, q_{1}, K^{m}\right)$ correspond to the intersection points of $\varphi_{K}^{m}\left(T_{q_{0}}^{*} M\right)$ and $T_{q_{1}}^{*} M$ via the evaluation map $x \mapsto x(1)$.

Fix now $q_{0} \in M$, and let $V\left(q_{0}, K^{m}\right)$ be the set of those $q_{1} \in M$ for which $\varphi_{K}^{m}\left(D_{q_{0}}\left(r_{H}+2 \epsilon\right)\right)$ and $D_{q_{1}}\left(r_{H}+2 \epsilon\right)$ intersect transversely.

Lemma 2.1. The set $V\left(q_{0}, K^{m}\right)$ is open and of full measure in $M$.

Proof. Since $D_{q_{0}}\left(r_{H}+2 \epsilon\right)$ is compact, $V\left(q_{0}, K^{m}\right)$ is open. Applying Sard's theorem to the projection $\varphi_{K}^{m}\left(D_{q_{0}}\left(r_{H}+2 \epsilon\right)\right) \rightarrow M$, one sees that $V\left(q_{0}, K^{m}\right)$ has full measure in $M$.

Consider the "annuli-bundle"

$$
A(\epsilon)=\left\{(q, p) \in T^{*} M\left|r_{H}+\epsilon \leq\right| p \mid \leq r_{H}+2 \epsilon\right\},
$$

set $A_{q_{0}}(\epsilon)=A(\epsilon) \cap T_{q_{0}}^{*} M$, and let $W\left(q_{0}, K^{m}\right)$ be the set of those $q_{1} \in$ $V\left(q_{0}, K^{m}\right)$ for which $\varphi_{K}^{m}\left(A_{q_{0}}(\epsilon)\right) \cap A_{q_{1}}(\epsilon)$ is empty. For $q_{1} \in W\left(q_{0}, K^{m}\right)$, the set

$$
\mathcal{P}\left(q_{0}, q_{1}, K^{m}, r_{H}+2 \epsilon\right):=\left\{x \in \mathcal{P}\left(q_{0}, q_{1}, K^{m}\right) \mid x \subset D\left(r_{H}+2 \epsilon\right)\right\}
$$

is finite and contained in $D\left(r_{H}+\epsilon\right)$. In view of the Lagrangian foliation given by the fibers of $T^{*} M$, the Maslov index $\mu(x)$ of each $x \in$ $\mathcal{P}\left(q_{0}, q_{1}, K^{m}, r_{H}+2 \epsilon\right)$ is a well-defined integer, which in case of a geodesic Hamiltonian agrees with the Morse index of the corresponding geodesic path, see $[\mathbf{1}$, Section 1.2]. 
Lagrangian Floer homology. Floer homology for Lagrangian intersections was invented by Floer in a series of seminal papers, [23-26]. We shall use a version of Lagrangian Floer homology described in $[\mathbf{3 0}, \mathbf{4 1}]$, with the only add on that we work over arbitrary coefficient fields $\mathbb{F}_{p}$ instead of $\mathbb{F}_{2}$ only. In the above situation, we define the $k$ th Floer chain group $\mathrm{CF}_{k}\left(q_{0}, q_{1}, K^{m}, r_{H}+2 \epsilon ; \mathbb{F}_{p}\right)$ as the finite-dimensional $\mathbb{F}_{p}$-vector space freely generated by the elements of $\mathcal{P}\left(q_{0}, q_{1}, K^{m}, r_{H}+2 \epsilon\right)$ of Maslov index $k$ and the full Floer chain group as

$$
\mathrm{CF}_{*}\left(q_{0}, q_{1}, K^{m}, r_{H}+2 \epsilon ; \mathbb{F}_{p}\right)=\bigoplus_{k \in \mathbb{Z}} \mathrm{CF}_{k}\left(q_{0}, q_{1}, K^{m}, r_{H}+2 \epsilon ; \mathbb{F}_{p}\right) .
$$

In order to define the Floer boundary operator, we follow $[\mathbf{9}, \mathbf{1 4}, \mathbf{7 2}]$ and consider the non-empty and connected set $\mathcal{J}$ of $t$-dependent smooth families $\mathbf{J}=\left\{J_{t}\right\}, t \in[0,1]$, of $\omega$-compatible almost complex structures on $D\left(r_{H}+2 \epsilon\right)$ such that $J_{t}$ is convex and independent of $t$ on $A(\epsilon)$. This particularly means that $J$ is invariant under the local flow of the Liouville vector field $Y=\sum p_{j} \partial p_{j}$ on $A(\epsilon)$. For $\mathbf{J} \in \mathcal{J}$, for smooth maps $u$ from the strip $S=\mathbb{R} \times[0,1]$ to $D\left(r_{H}+2 \epsilon\right)$, and for $x^{ \pm} \in \mathcal{P}\left(q_{0}, q_{1}, K^{m}, r_{H}+2 \epsilon\right)$, consider Floer's equation

$$
\left\{\begin{array}{l}
\partial_{s} u+J_{t}(u)\left(\partial_{t} u-X_{K_{t}^{m}}(u)\right)=0 \\
u(s, j) \in T_{q_{j}}^{*} M, \quad j=0,1, \\
\lim _{s \rightarrow \pm \infty} u(s, t)=x^{ \pm}(t) \quad \text { uniformly in } t .
\end{array}\right.
$$

Lemma 2.2. Solutions of (2.2) are contained in $D\left(r_{H}+\epsilon\right)$.

Sketch of proof. Since $q_{1} \in W\left(q_{0}, K^{m}\right)$,

$$
\lim _{s \rightarrow \pm \infty} u(s, t)=x^{ \pm}(t) \subset D\left(r_{H}+\epsilon\right) .
$$

In view of the strong maximum principle, the lemma follows from the convexity of $J$ on $A(\epsilon)$ and from (2.3) together with the fact that the special form (2.1) of $K$ on $A(\epsilon)$ implies $\omega\left(Y, J X_{K^{m}}\right)=0$, cf. [30, 41].

We denote the set of solutions of $(2.2)$ by $\mathcal{M}\left(x^{-}, x^{+}, K^{m}, r_{H}+2 \epsilon ; \mathbf{J}\right)$. Note that the group $\mathbb{R}$ freely acts on $\mathcal{M}\left(x^{-}, x^{+}, K^{m}, r_{H}+2 \epsilon ; \mathbf{J}\right)$ by timeshift. Lemma 2.2 is an important ingredient to establish the compactness of the quotients $\mathcal{M}\left(x^{-}, x^{+}, K^{m}, r_{H}+2 \epsilon ; \mathbf{J}\right) / \mathbb{R}$. The other ingredient is that there is no bubbling-off of $\mathbf{J}$-holomorphic spheres or discs. Indeed, $[\omega]$ vanishes on $\pi_{2}\left(T^{*} M\right)$ because $\omega=d \lambda$ is exact, and $[\omega]$ vanishes on $\pi_{2}\left(T^{*} M, T_{q_{j}}^{*} M\right)$ because $\lambda$ vanishes on $T_{q_{j}}^{*} M, j=0,1$. See, for instance, $[\mathbf{2 4}, \mathbf{6 2}]$ for more details.

There exists a generic subset $\mathcal{J}_{\text {reg }}$ of $\mathcal{J}$ such that for each $\mathbf{J} \in \mathcal{J}_{\text {reg }}$, the moduli space $\mathcal{M}\left(x^{-}, x^{+}, K^{m}, r_{H}+2 \epsilon ; \mathbf{J}\right)$ is a smooth manifold of dimension $\mu\left(x^{-}\right)-\mu\left(x^{+}\right)$for all $x^{ \pm} \in \mathcal{P}\left(q_{0}, q_{1}, K^{m}, r_{H}+2 \epsilon\right)$, see [27]. Fix $\mathbf{J} \in \mathcal{J}_{\text {reg. }}$. It 
is shown in $\left[\mathbf{1}\right.$, Section 1.4] that the manifolds $\mathcal{M}\left(x^{-}, x^{+}, K^{m}, r_{H}+2 \epsilon ; \mathbf{J}\right)$ can be oriented in a way which is coherent with gluing, so that with the usual definition of the Floer boundary operators

$$
\partial_{k}(\mathbf{J}): \mathrm{CF}_{k}\left(q_{0}, q_{1}, K^{m}, r_{H}+2 \epsilon ; \mathbb{F}_{p}\right) \longrightarrow \mathrm{CF}_{k-1}\left(q_{0}, q_{1}, K^{m}, r_{H}+2 \epsilon ; \mathbb{F}_{p}\right)
$$

one finds $\partial_{k-1}(\mathbf{J}) \circ \partial_{k}(\mathbf{J})=0$ for each $k$. The proof makes use of the compactness of the zero- and one-dimensional components of $\mathcal{M}\left(x^{-}, x^{+}, K^{m}\right.$, $\left.r_{H}+2 \epsilon ; \mathbf{J}\right) / \mathbb{R}$, see $[\mathbf{1}, \mathbf{2 3}, \mathbf{6 4}]$. As our notation suggests, the Floer homology groups

$$
\mathrm{HF}_{k}\left(q_{0}, q_{1}, K^{m}, r_{H}+2 \epsilon ; \mathbb{F}_{p}\right):=\frac{\operatorname{ker} \partial_{k}(\mathbf{J})}{\operatorname{im} \partial_{k+1}(\mathbf{J})}
$$

do not depend on the choices involved in their construction: They neither depend on coherent orientations up to canonical isomorphisms, $[\mathbf{1}$, Section 1.7], nor do they depend on $\mathbf{J} \in \mathcal{J}_{\text {reg }}$ up to natural isomorphisms, as a continuation argument shows $[\mathbf{2 3}, \mathbf{6 4}]$. The same continuation argument also shows that the Floer homology groups do not alter if we add to $K^{m}$ a function supported in $[0,1] \times D\left(r_{H}+\epsilon\right)$. The function $G_{\beta}^{m}:=m \beta|p|^{2}$ is such a function so that

$$
\mathrm{HF}_{*}\left(q_{0}, q_{1}, K^{m}, r_{H}+2 \epsilon ; \mathbb{F}_{p}\right) \cong \mathrm{HF}_{*}\left(q_{0}, q_{1}, G_{\beta}^{m}, r_{H}+2 \epsilon ; \mathbb{F}_{p}\right),
$$

provided that $q_{1}$ also belongs to $V\left(q_{0}, G_{\beta}^{m}\right)$.

Remark 2.3. For most applications of Floer homology found so far, it suffices to work over the coefficient field $\mathbb{F}_{2}$. In view of Example 1.3 (c), it is important that we can work over arbitrary coefficient fields $\mathbb{F}_{p}$.

Proposition 2.4. Assume that $q_{1} \in W\left(q_{0}, G_{\beta}^{m}\right)$.

(i) If $(M, g)$ is energy hyperbolic, there exists $p \in \mathbb{P}$ such that

$$
\operatorname{dim} \mathrm{HF}_{*}\left(q_{0}, q_{1}, G_{\beta}^{m}, r_{H}+2 \epsilon ; \mathbb{F}_{p}\right) \geq \mathrm{e}^{\left(2 \beta r_{H} C(M, g)\right) m}
$$

for all large enough $m \in \mathbb{N}$.

(ii) In general, for every $\delta>0$, there exists $p \in \mathbb{P}$ such that

$$
\operatorname{dim} \mathrm{HF}_{*}\left(q_{0}, q_{1}, G_{\beta}^{m}, r_{H}+2 \epsilon ; \mathbb{F}_{p}\right) \geq m^{c(M)-\delta}
$$

for all large enough $m \in \mathbb{N}$.

Proposition 2.4 will be proved in the next step. In the remainder of this step, we show

Proposition 2.4 $\Longrightarrow$ Theorem 1.2. We show that Proposition 2.4(i) implies Theorem $1.2(\mathrm{i})$. Assume that $(M, g)$ is energy hyperbolic with exponent $C(M, g)$. Let $K$ be as before, and pick $q_{1} \in W\left(q_{0}, K^{m}\right) \cap$ $W\left(q_{0}, G_{\beta}^{m}\right)$. Since the generators of $\mathrm{CF}_{*}\left(q_{0}, q_{1}, K^{m}, r_{H}+2 \epsilon ; \mathbb{F}_{p}\right)$ correspond 
to $\varphi_{K}^{m}\left(D_{q_{0}}\left(r_{H}+\epsilon\right)\right) \cap D_{q_{1}}\left(r_{H}+\epsilon\right)$, we find together with (2.4) and Proposition 2.4 (i) that

$$
\#\left(\varphi_{K}^{m}\left(D_{q_{0}}\left(r_{H}+\epsilon\right)\right) \cap D_{q_{1}}\left(r_{H}+\epsilon\right)\right)=\operatorname{dim} \mathrm{CF}_{*}\left(q_{0}, q_{1}, K^{m}, r_{H}+2 \epsilon ; \mathbb{F}_{p}\right)
$$

$$
\begin{aligned}
& \geq \operatorname{dim} \mathrm{HF}_{*}\left(q_{0}, q_{1}, K^{m}, r_{H}+2 \epsilon ; \mathbb{F}_{p}\right) \\
& =\operatorname{dim} \mathrm{HF}_{*}\left(q_{0}, q_{1}, G_{\beta}^{m}, r_{H}+2 \epsilon ; \mathbb{F}_{p}\right) \\
& \geq \mathrm{e}^{\left(2 \beta r_{H} C(M, g)\right) m}
\end{aligned}
$$

for some $p \in \mathbb{P}$ and for $m$ large enough. Recall now that $\beta=f^{\prime}\left(r_{H}\right)$. We thus find a sequence $\epsilon_{i} \rightarrow 0$ and a sequence $K_{i}:[0,1] \times T^{*} M \rightarrow \mathbb{R}$ of $C^{\infty}$-smooth functions such that

$$
K_{i}(t, q, p)=\beta|p|^{2} \quad \text { if }|p| \geq r_{H}+\epsilon_{i}
$$

and such that

(K1) $\left.K_{i}\right|_{D\left(r_{H}+\epsilon_{i}\right)}$ is uniformly bounded in the $C^{2}$-topology;

$\left.\left.(\mathrm{K} 2) K_{i}\right|_{D\left(r_{H}\right)} \rightarrow H\right|_{D\left(r_{H}\right)}$ in the $C^{2}$-topology.

Note that $\pi: T^{*} M \rightarrow M$ is a Riemannian submersion with respect to the Riemannian metrics $g^{*}$ and $g$. Applying (2.5) to $K_{i}$, we therefore find

$$
\begin{aligned}
& \mu_{g^{*}}\left(\varphi_{K_{i}}^{m}\left(D_{q_{0}}\left(r_{H}+\epsilon_{i}\right)\right)\right) \\
& \quad \geq \mathrm{e}^{\left(2 \beta r_{H} C(M, g)\right) m} \mu_{g}\left(W\left(q_{0}, K_{i}^{m}\right) \cap W\left(q_{0}, G_{\beta}^{m}\right)\right) .
\end{aligned}
$$

Since $\epsilon_{i} \rightarrow 0$, we have

$$
\lim _{i \rightarrow \infty} \mu_{g^{*}}\left(\varphi_{K_{i}}^{m}\left(A\left(\epsilon_{i}\right)\right)\right)=\lim _{i \rightarrow \infty} \mu_{g^{*}}\left(\varphi_{G_{\beta}}^{m}\left(A\left(\epsilon_{i}\right)\right)\right)=0,
$$

so that, together with Lemma 2.1,

$$
\begin{aligned}
\lim _{i \rightarrow \infty} \mu_{g}\left(W\left(q_{0}, K_{i}^{m}\right) \cap W\left(q_{0}, G_{\beta}^{m}\right)\right) & =\lim _{i \rightarrow \infty} \mu_{g}\left(V\left(q_{0}, K_{i}^{m}\right) \cap V\left(q_{0}, G_{\beta}^{m}\right)\right) \\
& =\mu_{g}(M) .
\end{aligned}
$$

Moreover, $\epsilon_{i} \rightarrow 0$ and (K1) imply

$$
\lim _{i \rightarrow \infty} \mu_{g^{*}}\left(\varphi_{K_{i}}^{m}\left(D_{q_{0}}\left(r_{H}+\epsilon_{i}\right) \backslash D_{q_{0}}\left(r_{H}\right)\right)\right)=0,
$$

and (K2) implies

$$
\lim _{i \rightarrow \infty} \mu_{g^{*}}\left(\varphi_{K_{i}}^{m}\left(D_{q_{0}}\left(r_{H}\right)\right)\right)=\mu_{g^{*}}\left(\varphi_{H}^{m}\left(D_{q_{0}}\left(r_{H}\right)\right)\right) .
$$

Using (2.9), (2.8), (2.6), and (2.7), we find that

$$
\mu_{g^{*}}\left(\varphi_{H}^{m}\left(D_{q_{0}}\left(r_{H}\right)\right)\right) \geq \mathrm{e}^{\left(2 \beta r_{H} C(M, g)\right) m} \mu_{g}(M)
$$

for $m$ large enough. Since $q_{0} \in M$ was arbitrary, this yields $\check{v}_{\text {fibre }}\left(\varphi_{H} ; r_{H}\right) \geq$ $2 \beta r_{H} C(M, g)$, as claimed.

In a similar way, Proposition 2.4 (ii) implies that in general $\check{s}_{\text {fibre }}\left(\varphi_{H} ; r_{H}\right)$ $\geq c(M)-\delta$ for every $\delta>0$ and hence $\check{s}_{\text {fibre }}\left(\varphi_{H} ; r_{H}\right) \geq c(M)$. The estimate $c(M) \geq 1$ is guaranteed by Propositions 1.4 and 1.8 . 


\section{Step 2. From Floer homology to the homology of the path space}

In this step, we prove Proposition 2.4. Viterbo $[\mathbf{7 1}, \mathbf{7 2}]$ was the first to notice that the Floer homology for periodic orbits of $T^{*} M$ is isomorphic to the singular homology of the free loop space of $M$. Different proofs were found by Salamon-Weber [63] and Abbondandolo-Schwarz [1]. The work [1] also establishes a relative version of this result: the Floer homology for Lagrangian intersections of $T^{*} M$ is isomorphic to the singular homology of the based loop space of $M$. It is this version that we shall take advantage of.

We abbreviate $\rho=r_{H}+2 \epsilon$. In order to estimate the dimension of $\mathrm{HF}_{*}\left(q_{0}, q_{1}, G_{\beta}^{m}, \rho ; \mathbb{F}_{p}\right)$ from below, we first describe these $\mathbb{F}_{p}$-vector spaces in a somewhat different way. Set

$$
\mathcal{P}^{m \beta \rho^{2}}\left(q_{0}, q_{1}, G_{\beta}^{m}\right):=\left\{x \in \mathcal{P}\left(q_{0}, q_{1}, G_{\beta}^{m}\right) \mid \mathcal{A}_{G_{\beta}^{m}}(x) \leq m \beta \rho^{2}\right\} .
$$

Lemma 2.5. $\mathcal{P}^{m \beta \rho^{2}}\left(q_{0}, q_{1}, G_{\beta}^{m}\right)=\mathcal{P}\left(q_{0}, q_{1}, G_{\beta}^{m}, \rho\right)$.

Proof. Assume that $x \in \mathcal{P}\left(q_{0}, q_{1}, G_{\beta}^{m}\right)$. For $t_{0} \in[0,1]$, we choose geodesic normal coordinates $q$ near $\pi\left(x\left(t_{0}\right)\right)$ in $M$. With respect to these coordinates, the equation $\dot{x}=X_{G_{\beta}^{m}}(x)$ at $t_{0}$ reads

$$
\begin{aligned}
\dot{p}\left(t_{0}\right) & =0, \\
\dot{q}\left(t_{0}\right) & =2 m \beta p\left(t_{0}\right) .
\end{aligned}
$$

Therefore, $\lambda\left(\dot{x}\left(t_{0}\right)\right)-G_{\beta}^{m}\left(x\left(t_{0}\right)\right)=2 m \beta|p|^{2}-m \beta|p|^{2}=m \beta|p|^{2}=G_{\beta}^{m}\left(x\left(t_{0}\right)\right)$. Since $G_{\beta}^{m}$ is autonomous, $G_{\beta}^{m}\left(x\left(t_{0}\right)\right)$ does not depend on $t_{0}$. Integrating over $[0,1]$, we thus obtain $\mathcal{A}_{G_{\beta}^{m}}(x)=G_{\beta}^{m}(x)$. The lemma follows.

We define the $k$ th Floer chain group $\mathrm{CF}_{k}^{m \beta \rho^{2}}\left(q_{0}, q_{1}, G_{\beta}^{m} ; \mathbb{F}_{p}\right)$ as the finite-dimensional $\mathbb{F}_{p}$-vector space freely generated by the elements of $\mathcal{P}^{m \beta \rho^{2}}\left(q_{0}, q_{1}, G_{\beta}^{m}\right)$ of Maslov index $k$. Lemma 2.5 yields

Lemma 2.6. $\mathrm{CF}_{*}^{m \beta \rho^{2}}\left(q_{0}, q_{1}, G_{\beta}^{m} ; \mathbb{F}_{p}\right)=\mathrm{CF}_{*}\left(q_{0}, q_{1}, G_{\beta}^{m}, \rho ; \mathbb{F}_{p}\right)$.

Denote by $\widehat{\mathcal{J}}$ the set of families $\widehat{\mathbf{J}}=\left\{\widehat{J}_{t}\right\}$ of almost complex structures on $T^{*} M$ such that $\left.\widehat{\mathbf{J}}\right|_{D(\rho)} \in \mathcal{J}$ and $\widehat{\mathbf{J}}$ is invariant under the flow of $Y$ on $T^{*} M \backslash D\left(r_{H}+\epsilon\right)$. For $\widehat{\mathbf{J}} \in \widehat{\mathcal{J}}$, for smooth maps $u: S \rightarrow T^{*} M$, and for $x^{ \pm} \in \mathcal{P}^{m \beta \rho^{2}}\left(q_{0}, q_{1}, G_{\beta}^{m}\right)$ consider Floer's equation

$$
\left\{\begin{array}{l}
\partial_{s} u+\widehat{J}_{t}(u)\left(\partial_{t} u-X_{G_{\beta}^{m}}(u)\right)=0, \\
u(s, j) \in T_{q_{j}}^{*} M, \quad j=0,1, \\
\lim _{s \rightarrow \pm \infty} u(s, t)=x^{ \pm}(t) \quad \text { uniformly in } t .
\end{array}\right.
$$

We denote the set of solutions of $(2.10)$ by $\mathcal{M}^{m \beta \rho^{2}}\left(x^{-}, x^{+}, G_{\beta}^{m} ; \widehat{\mathbf{J}}\right)$. Lemma 2.5 and 2.2 imply 
Lemma 2.7. $\mathcal{M}^{m \beta \rho^{2}}\left(x^{-}, x^{+}, G_{\beta}^{m} ; \widehat{\mathbf{J}}\right)=\mathcal{M}\left(x^{-}, x^{+}, G_{\beta}^{m}, \rho ; \mathbf{J}\right)$.

A standard argument shows that $\mathcal{A}_{G_{\beta}^{m}}\left(x^{-}\right) \geq \mathcal{A}_{G_{\beta}^{m}}\left(x^{+}\right)$for each $u \in$ $\mathcal{M}^{m \beta \rho^{2}}\left(x^{-}, x^{+}, G_{\beta}^{m} ; \widehat{\mathbf{J}}\right)$. For generic $\widehat{\mathbf{J}} \in \widehat{\mathcal{J}}$, the usual definition of the Floer boundary operator therefore yields boundary operators

$$
\partial_{k}(\widehat{\mathbf{J}}): \mathrm{CF}_{k}^{m \beta \rho^{2}}\left(q_{0}, q_{1}, G_{\beta}^{m} ; \mathbb{F}_{p}\right) \longrightarrow \mathrm{CF}_{k-1}^{m \beta \rho^{2}}\left(q_{0}, q_{1}, G_{\beta}^{m} ; \mathbb{F}_{p}\right) .
$$

As before, their homology groups $\mathrm{HF}_{k}^{m \beta \rho^{2}}\left(q_{0}, q_{1}, G_{\beta}^{m} ; \mathbb{F}_{p}\right)$ neither depend on coherent orientations of $\mathcal{M}^{m \beta \rho^{2}}\left(x^{-}, x^{+}, G_{\beta}^{m} ; \widehat{\mathbf{J}}\right)$ nor on $\widehat{\mathbf{J}}$. Lemmata 2.6 and 2.7 imply that $\partial_{k}(\widehat{\mathbf{J}})=\partial_{k}(\mathbf{J})$, whence

Proposition 2.8. $\mathrm{HF}_{*}\left(q_{0}, q_{1}, G_{\beta}^{m}, \rho ; \mathbb{F}_{p}\right) \cong \mathrm{HF}_{*}^{m \beta \rho^{2}}\left(q_{0}, q_{1}, G_{\beta}^{m} ; \mathbb{F}_{p}\right)$.

For $q_{0}, q_{1} \in M$, let $\Omega^{1}\left(M, q_{0}, q_{1}\right)$ be the space of all paths $q:[0,1] \rightarrow M$ of Sobolev class $W^{1,2}$ such that $q(0)=q_{0}$ and $q(1)=q_{1}$. Again, this space has a canonical Hilbert manifold structure. The energy functional $\mathcal{E}: \Omega^{1}\left(M, q_{0}, q_{1}\right) \rightarrow \mathbb{R}$ is defined as

$$
\mathcal{E}(q)=\frac{1}{2} \int_{0}^{1}|\dot{q}(t)|^{2} d t
$$

For $a>0$, we consider the sublevel sets

$$
\mathcal{E}^{a}\left(q_{0}, q_{1}\right):=\left\{q \in \Omega^{1}\left(M, q_{0}, q_{1}\right) \mid \mathcal{E}(q) \leq a\right\} .
$$

Proposition 2.9. $\mathrm{HF}_{*}^{m \beta \rho^{2}}\left(q_{0}, q_{1}, G_{\beta}^{m} ; \mathbb{F}_{p}\right) \cong \mathrm{H}_{*}\left(\mathcal{E}^{2(\beta \rho m)^{2}}\left(q_{0}, q_{1}\right) ; \mathbb{F}_{p}\right)$.

Proof. Let $L: T M \rightarrow \mathbb{R}$ be the Legendre transform of $G_{\beta}^{m}$. Applying Theorem 3.1 of $[\mathbf{1}]$ to $G_{\beta}^{m}$ and $L$, we obtain

$$
\begin{aligned}
& \mathrm{HF}_{*}^{m \beta \rho^{2}}\left(q_{0}, q_{1}, G_{\beta}^{m} ; \mathbb{F}_{p}\right) \\
& \quad \cong \mathrm{H}_{*}\left(\left\{q \in \Omega^{1}\left(M, q_{0}, q_{1}\right) \mid \int_{0}^{1} L(q(t), \dot{q}(t)) d t \leq m \beta \rho^{2}\right\} ; \mathbb{F}_{p}\right) .
\end{aligned}
$$

Notice now that $L(q, v)=1 /(4 m \beta)|v|^{2}$. The set $\{\cdots\}$ on the right hand side therefore equals $\mathcal{E}^{2(\beta \rho m)^{2}}\left(q_{0}, q_{1}\right)$, and so Proposition 2.9 follows.

Remark 2.10. Strictly speaking, Abbondandolo and Schwarz work in [1] with almost complex structures which are close to the almost complex structure interchanging the horizontal and vertical tangent bundles of $\left(T^{*} M, g^{*}\right)$. They need to work with such almost complex structures in order to prove a subtle $L^{\infty}$-estimate for solutions of Floer's equation below a given action which is crucial for obtaining their Theorem 3.1 for a large class of Hamiltonians. For the special Hamiltonians $G_{\beta}^{m}$ appearing in our situation, one can work with convex almost complex structures $\widehat{\mathbf{J}} \in \widehat{\mathcal{J}}$ and does not need 
their $L^{\infty}$-estimate, since boundedness of solutions of Floer's equation below a given action then follows from Lemma 2.7.

Propositions 2.8 and 2.9 yield

Proposition 2.11. $\mathrm{HF}_{*}\left(q_{0}, q_{1}, G_{\beta}^{m}, \rho ; \mathbb{F}_{p}\right) \cong \mathrm{H}_{*}\left(\mathcal{E}^{2(\beta \rho m)^{2}}\left(q_{0}, q_{1}\right) ; \mathbb{F}_{p}\right)$.

The dimension of this vector space is

$\sum_{k \geq 0} \operatorname{dim} \mathrm{H}_{k}\left(\mathcal{E}^{(1 / 2)(2 \beta \rho m)^{2}}\left(q_{0}, q_{1}\right) ; \mathbb{F}_{p}\right) \geq \sum_{k \geq 0} \operatorname{dim} \iota_{k} \mathrm{H}_{k}\left(\mathcal{E}^{(1 / 2)(2 \beta \rho m)^{2}}\left(q_{0}, q_{1}\right) ; \mathbb{F}_{p}\right)$,

and the growth in $m$ of the sequence on the right does not depend on $q_{0}$ and $q_{1}$. Since $\rho=r_{H}+2 \epsilon>r_{H}$, Proposition 2.4(i) now follows in view of the definition of $C(M, g)$, and Proposition 2.4 (ii) follows in view of the definition of $c(M)$.

2.2. Proof of Proposition 1.4. Consider a closed connected Riemannian manifold $(M, g)$ with finite $\pi_{1}(M)$. We first verify that we can suppose $M$ to be simply connected.

Lemma 2.12. Let $(\widetilde{M}, \tilde{g})$ be the universal cover of $(M, g)$.

(a) The manifold $M$ is elliptic resp. hyperbolic if and only if $\widetilde{M}$ is elliptic resp. hyperbolic, and $\rho(M)=\rho(\widetilde{M})$.

(b) The Riemannian manifold $(M, g)$ is energy hyperbolic if and only if $(\widetilde{M}, \tilde{g})$ is energy hyperbolic, in which case $C(M, g)=C(\widetilde{M}, \tilde{g})$. In general, $c(M)=c(\widetilde{M})$.

Proof. We only give a proof of $C(M, g)=C(\widetilde{M}, \tilde{g})$, since it essentially contains a proof of the other assertions. Choose a point $\tilde{q}_{0} \in \widetilde{M}$ over $q_{0} \in$ $M$. The map $\Omega^{1}(\pi): \Omega^{1}\left(\widetilde{M}, \tilde{q}_{0}\right) \rightarrow \Omega^{1}\left(M, q_{0}\right)$ induced by the projection $\pi: \widetilde{M} \rightarrow M$ maps $\Omega^{1}\left(\widetilde{M}, \tilde{q}_{0}\right)$ homeomorphically onto the component $\mathcal{C}_{1}$ of the constant loop $\gamma_{1} \equiv q_{0}$; since $\tilde{g}$ is the lift of $g$, it in fact maps each $\mathcal{E}^{a}\left(\tilde{q}_{0}\right)$ homeomorphically onto $\mathcal{E}^{a}\left(q_{0}\right) \cap \mathcal{C}_{1}$. Therefore, $C(\widetilde{M}, \tilde{g}) \leq C(M, g)$. In order to prove the reverse inequality, we choose in each other component $\mathcal{C}_{i}$ of $\Omega^{1}\left(M, q_{0}\right)$ a loop $\gamma_{i}, i=2, \ldots, l=\# \pi_{1}(M)$. Fix $\epsilon \in(0,1)$. For $\gamma, \gamma^{\prime} \in \Omega^{1}\left(M, q_{0}\right)$, define $\gamma *_{\epsilon} \gamma^{\prime} \in \Omega^{1}\left(M, q_{0}\right)$ by

$$
\left(\gamma *_{\epsilon} \gamma^{\prime}\right)(t)= \begin{cases}\gamma\left(\frac{t}{\epsilon}\right), & 0 \leq t \leq \epsilon, \\ \gamma^{\prime}\left(\frac{t-\epsilon}{1-\epsilon}\right), & \epsilon \leq t \leq 1 .\end{cases}
$$

It is easy to see that the $\operatorname{map} \mathcal{C}_{1} \rightarrow \mathcal{C}_{i}, \gamma \mapsto \gamma_{i} *_{\epsilon} \gamma$, is a homotopy equivalence with homotopy inverse $\Gamma_{i}: \mathcal{C}_{i} \rightarrow \mathcal{C}_{1}, \gamma \mapsto \gamma_{i}^{-1} *_{\epsilon} \gamma$. Notice that

$$
\mathcal{E}\left(\gamma *_{\epsilon} \gamma^{\prime}\right)=\frac{1}{\epsilon} \mathcal{E}(\gamma)+\frac{1}{1-\epsilon} \mathcal{E}\left(\gamma^{\prime}\right) \quad \text { for all } \gamma, \gamma^{\prime} \in \Omega^{1}\left(M, q_{0}\right)
$$


Abbreviating $\mathcal{E}_{i}^{a}\left(q_{0}\right)=\mathcal{E}^{a}\left(q_{0}\right) \cap \mathcal{C}_{i}$ and $E=\max \left\{\mathcal{E}\left(\gamma_{i}^{-1}\right) \mid i=2, \ldots, l\right\}$, we therefore have

$$
\Gamma_{i}\left(\mathcal{E}_{i}^{a}\left(q_{0}\right)\right) \subset \mathcal{E}_{1}^{a /(1-\epsilon)+E / \epsilon}\left(q_{0}\right) .
$$

Since $\Gamma_{i}: \mathcal{C}_{i} \rightarrow \mathcal{C}_{1}$ is a homotopy equivalence, it follows that

$$
\begin{aligned}
\operatorname{dim} \iota_{k} \mathrm{H}_{k}\left(\mathcal{E}_{i}^{a}\left(q_{0}\right) ; \mathbb{F}_{p}\right) & =\operatorname{dim} \iota_{k} \mathrm{H}_{k}\left(\Gamma_{i}\left(\mathcal{E}_{i}^{a}\left(q_{0}\right)\right) ; \mathbb{F}_{p}\right) \\
& \leq \operatorname{dim} \iota_{k} \mathrm{H}_{k}\left(\mathcal{E}_{1}^{a /(1-\epsilon)+E / \epsilon}\left(q_{0}\right) ; \mathbb{F}_{p}\right) \\
& =\operatorname{dim} \iota_{k} \mathrm{H}_{k}\left(\mathcal{E}^{a /(1-\epsilon)+E / \epsilon}\left(\tilde{q}_{0}\right) ; \mathbb{F}_{p}\right) .
\end{aligned}
$$

Therefore,

$$
\begin{aligned}
\operatorname{dim} \iota_{k} \mathrm{H}_{k}\left(\mathcal{E}^{a}\left(q_{0}\right) ; \mathbb{F}_{p}\right) & =\sum_{i=1}^{l} \operatorname{dim} \iota_{k} \mathrm{H}_{k}\left(\mathcal{E}_{i}^{a}\left(q_{0}\right) ; \mathbb{F}_{p}\right) \\
& \leq l \operatorname{dim} \iota_{k} \mathrm{H}_{k}\left(\mathcal{E}^{a /(1-\epsilon)+E / \epsilon}\left(\tilde{q}_{0}\right) ; \mathbb{F}_{p}\right),
\end{aligned}
$$

and so

$$
\begin{aligned}
& \liminf _{m \rightarrow \infty} \frac{1}{m} \log \sum_{k \geq 0} \operatorname{dim} \iota_{k} \mathrm{H}_{k}\left(\mathcal{E}^{(1 / 2) m^{2}}\left(q_{0}\right) ; \mathbb{F}_{p}\right) \\
& \leq \liminf _{m \rightarrow \infty} \frac{1}{m} \log \sum_{k \geq 0} l \operatorname{dim} \iota_{k} \mathrm{H}_{k}\left(\mathcal{E}^{(1 / 2)(m / \sqrt{1-\epsilon})^{2}+E / \epsilon}\left(\tilde{q}_{0}\right) ; \mathbb{F}_{p}\right) \\
& \quad=\frac{1}{\sqrt{1-\epsilon}} \liminf _{m \rightarrow \infty} \frac{1}{m} \log \sum_{k \geq 0} \operatorname{dim} \iota_{k} \mathrm{H}_{k}\left(\mathcal{E}^{(1 / 2) m^{2}}\left(\tilde{q}_{0}\right) ; \mathbb{F}_{p}\right) .
\end{aligned}
$$

Since this is true for all $p \in \mathbb{P}$, we obtain $C(M, g) \leq 1 / \sqrt{1-\epsilon} C(\widetilde{M}, \tilde{g})$, and since $\epsilon \in(0,1)$ was arbitrary, we conclude that $C(M, g) \leq C(\widetilde{M}, \tilde{g})$.

In view of Lemma 2.12 , we can assume that $(M, g)$ is simply connected throughout the proof of Proposition 1.4.

We next prove the implication " $M$ hyperbolic $\Rightarrow(M, g)$ energy hyperbolic" and the inequalities $c(M) \geq \rho(M) \geq 1$. The length functional $\mathcal{L}=$ $\mathcal{L}_{g}: \Omega^{1}\left(M, q_{0}\right) \rightarrow \mathbb{R}$ is defined as

$$
\mathcal{L}(q)=\int_{0}^{1}|\dot{q}(t)| d t
$$

For $a>0$, we consider the sublevel sets $\mathcal{L}^{a}\left(q_{0}\right):=\left\{q \in \Omega^{1}\left(M, q_{0}\right) \mid \mathcal{L}(q) \leq a\right\}$.

Lemma 2.13. There exists a constant $C_{G}>0$ depending only on $(M, g)$ such that each element of $\mathrm{H}_{k}\left(\Omega^{1}\left(M, q_{0}\right) ; \mathbb{F}_{p}\right)$ can be represented by a cycle in $\mathcal{E}^{(1 / 2)\left(C_{G} k\right)^{2}}\left(q_{0}\right)$. In particular,

$\sum_{k \geq 0} \operatorname{dim} \iota_{k} \mathrm{H}_{k}\left(\mathcal{E}^{(1 / 2)\left(C_{G} m\right)^{2}}\left(q_{0}\right) ; \mathbb{F}_{p}\right) \geq \sum_{k=0}^{m} \operatorname{dim} \mathrm{H}_{k}\left(\Omega^{1}\left(M, q_{0}\right) ; \mathbb{F}_{p}\right) \quad$ for all $m$. 
Proof. According to a result of Gromov, there exists a constant $C_{G}>0$ depending only on $(M, g)$ such that each element of $\mathrm{H}_{k}\left(\Omega^{1}\left(M, q_{0}\right) ; \mathbb{F}_{p}\right)$ can be represented by a cycle lying in $\mathcal{L}^{\left(C_{G}-2\right) k}\left(q_{0}\right)$. Gromov's original proof of this result in [33] is very short. Detailed proofs can be found in [55] and $[\mathbf{3 5}$, Chapter $7 \mathrm{~A}]$. Let $\Delta^{k}$ be the $k$-dimensional standard simplex, and let $\psi=\sum_{i} n_{i} \psi^{i}: \Delta^{k} \rightarrow \mathcal{L}^{\left(C_{G}-2\right) k}\left(q_{0}\right)$ be an integral cycle. For notational convenience, we pretend that $\psi$ consists of only one simplex. By suitably reparametrizing each path $\psi(s)$ near $t=0$ and $t=1$ and then smoothing each path with the same heat kernel, we obtain a homotopic and hence homologous cycle $\psi_{1}: \Delta^{k} \rightarrow \mathcal{L}^{\left(C_{G}-1\right) k}\left(q_{0}\right)$ consisting of smooth paths. We identify $\psi_{1}$ with the map

$$
\Delta^{k} \times[0,1] \longrightarrow M, \quad(s, t) \longmapsto \psi_{1}(s, t):=\left(\psi_{1}(s)\right)(t) .
$$

Endow the manifold $M \times[0,1]$ with the product Riemannian metric, and set $\tilde{q}_{0}=\left(q_{0}, 0\right)$. We lift $\psi_{1}$ to the cycle $\widetilde{\psi}_{1}: \Delta^{k} \rightarrow \Omega^{1}\left(M \times[0,1], \tilde{q}_{0}\right)$ defined by $\widetilde{\psi}_{1}(s, t)=\left(\psi_{1}(s, t), t\right)$. This cycle consists of smooth paths whose tangent vectors do not vanish. For each $s$, let $\widetilde{\psi}_{1}(\sigma(s))$ be the reparametrization of $\widetilde{\psi}_{1}(s)$ proportional to arc length. The homotopy $\Psi:[0,1] \times \Delta^{k} \rightarrow \Omega^{1}\left(M \times[0,1], \tilde{q}_{0}\right)$ defined by

$$
(\Psi(\tau, s))(t)=\widetilde{\psi}_{1}(s,(1-\tau) t+\tau \sigma(s))
$$

shows that $\widetilde{\psi}_{1}$ is homologous to the cycle $\widetilde{\psi}_{2}(s):=\Psi(1, s)$. Its projection $\psi_{2}$ to $\Omega^{1}\left(M, q_{0}\right)$ is homologous to $\psi_{1}$ and lies in $\mathcal{L}^{\left(C_{G}-1\right) k}\left(q_{0}\right)$. Since for each $s$ the path $\widetilde{\psi}_{2}(s)$ is parametrized proportional to arc length, we conclude that

$$
\begin{aligned}
\mathcal{E}\left(\psi_{2}(s)\right) \leq \mathcal{E}\left(\widetilde{\psi}_{2}(s)\right) & =\frac{1}{2}\left(\mathcal{L}\left(\widetilde{\psi}_{2}(s)\right)\right)^{2} \\
& =\frac{1}{2}\left\{\left(\mathcal{L}\left(\psi_{2}(s)\right)\right)^{2}+1\right\} \\
& \leq \frac{1}{2}\left(C_{G}-1\right)^{2} k^{2}+\frac{1}{2} \leq \frac{1}{2}\left(C_{G} k\right)^{2}
\end{aligned}
$$

for each $s$, so that indeed $\psi_{2} \subset \mathcal{E}^{(1 / 2)\left(C_{G} k\right)^{2}}\left(q_{0}\right)$.

Recall that $\Omega\left(M, q_{0}\right)$ is the space of continuous paths $q:[0,1] \rightarrow M$ with $q(0)=q(1)=q_{0}$ endowed with the compact open topology. According to [51, Chapter 17] or [43, Theorem 1.2.10], the inclusion $\Omega^{1}\left(M, q_{0}\right) \rightarrow$ $\Omega\left(M, q_{0}\right)$ is a homotopy equivalence. The homotopy type of these spaces does not depend on $q_{0}$ and is denoted $\Omega(M)$. Together with Lemma 2.13, we find

$$
\begin{aligned}
\sum_{k \geq 0} \operatorname{dim} \iota_{k} \mathrm{H}_{k}\left(\mathcal{E}^{(1 / 2)\left(C_{G} m\right)^{2}}\left(q_{0}\right) ; \mathbb{F}_{p}\right) & \geq \sum_{k=0}^{m} \operatorname{dim} \mathrm{H}_{k}\left(\Omega(M) ; \mathbb{F}_{p}\right) \\
& =r_{m}\left(M ; \mathbb{F}_{p}\right)
\end{aligned}
$$


for all $m \in \mathbb{N}$ and $p \in \mathbb{P}$. It follows that $(M, g)$ is energy hyperbolic if $M$ is hyperbolic and that $c(M) \geq \rho(M)$. The estimate $\rho(M) \geq 1$ follows from Proposition 11 of Serre's seminal work [67, p. 483]. Applied to our closed simply connected manifold $M$ of dimension $d$, it guarantees that for every integer $i \geq 0$, there exists an integer $j \in\{1, \ldots, d-1\}$ such that $\mathrm{H}_{i+j}\left(\Omega(M) ; \mathbb{F}_{p}\right) \neq 0$.

We finally prove the implication " $(M, g)$ energy hyperbolic $\Rightarrow M$ hyperbolic" and the inequality $c(M) \leq \rho(M)$. Choose $\operatorname{conv}(M, g)>0$ so small that for each $q \in M$, the ball of radius $\operatorname{conv}(M, g)$ centered at $q$ is geodesically convex. According to the proof of Theorem 3.4 in [5],

$$
\mathrm{H}_{k}\left(\mathcal{E}^{a}\left(q_{0}\right) ; \mathbb{F}_{p}\right)=0 \quad \text { if } k>d\left\lfloor\frac{\sqrt{a}}{\operatorname{conv}(M, g)}+1\right\rfloor
$$

for all $a>0, q_{0} \in M$, and $p \in \mathbb{P}$. Here, $\lfloor r\rfloor=\max \{n \in \mathbb{Z} \mid n \leq r\}$. We therefore find a constant $D$ such that

$$
\begin{aligned}
\sum_{k \geq 0} \operatorname{dim} \iota_{k} \mathrm{H}_{k}\left(\mathcal{E}^{(1 / 2) m^{2}}\left(q_{0}\right) ; \mathbb{F}_{p}\right) & =\sum_{k=0}^{D m} \operatorname{dim} \iota_{k} \mathrm{H}_{k}\left(\mathcal{E}^{(1 / 2) m^{2}}\left(q_{0}\right) ; \mathbb{F}_{p}\right) \\
& \leq \sum_{k=0}^{D m} \operatorname{dim} \mathrm{H}_{k}\left(\Omega(M) ; \mathbb{F}_{p}\right)
\end{aligned}
$$

for all $m$, from which the two claims follow.

2.3. Proof of Proposition 1.8. Consider a closed connected Riemannian manifold $(M, g)$ with infinite $\pi_{1}(M)$. The starting point of the proof is the crude estimate

$$
\sum_{k \geq 0} \operatorname{dim} \iota_{k} \mathrm{H}_{k}\left(\mathcal{E}^{a}\left(q_{0}\right) ; \mathbb{F}_{p}\right) \geq \operatorname{dim} \iota_{0} \mathrm{H}_{0}\left(\mathcal{E}^{a}\left(q_{0}\right) ; \mathbb{F}_{p}\right) .
$$

Denote by $\Pi_{\mathcal{E}}^{a}\left(q_{0}\right)$ (resp. $\Pi_{\mathcal{L}}^{a}\left(q_{0}\right)$ ) the set of those homotopy classes of $W^{1,2}$-paths $q:[0,1] \rightarrow M$ with $q(0)=q(1)=q_{0}$ which can be represented by a path of energy (resp. length) at most $a$. The vector space $\iota_{0} \mathrm{H}_{0}\left(\mathcal{E}^{a}\left(q_{0}\right) ; \mathbb{F}_{p}\right)$ is freely generated by the elements of $\Pi_{\mathcal{E}}^{a}\left(q_{0}\right)$, i.e.,

$$
\operatorname{dim} \iota_{0} \mathrm{H}_{0}\left(\mathcal{E}^{a}\left(q_{0}\right) ; \mathbb{F}_{p}\right)=\# \Pi_{\mathcal{E}}^{a}\left(q_{0}\right) .
$$

Moreover,

$$
\# \Pi_{\mathcal{E}}^{(1 / 2) m^{2}}\left(q_{0}\right)=\# \Pi_{\mathcal{L}}^{m}\left(q_{0}\right) \quad \text { for all } m .
$$

Let now $S=\left\{h_{1}, \ldots, h_{\# S}\right\}$ be a generating set of $\pi_{1}(M)$. In view of the definition of $\ell(S, g)$ in Section 1.5, we can represent each $h_{j}$ by a smooth loop based at $q_{0}$ of length no more than $\ell(S, g)$. In view of the triangle inequality and the definition of the growth function $\gamma_{S}$, we obtain

$$
\# \Pi_{\mathcal{L}}^{m}\left(q_{0}\right) \geq \gamma_{S}\left(\left\lfloor\frac{m}{\ell(S, g)}\right\rfloor\right)
$$


where again $\lfloor r\rfloor=\max \{n \in \mathbb{Z} \mid n \leq r\}$. Summarizing, we have

$$
\sum_{k \geq 0} \operatorname{dim} \iota_{k} \mathrm{H}_{k}\left(\mathcal{E}^{(1 / 2) m^{2}}\left(q_{0}\right) ; \mathbb{F}_{p}\right) \geq \gamma_{S}\left(\left\lfloor\frac{m}{\ell(S, g)}\right\rfloor\right)
$$

for all $m \in \mathbb{N}$ and all $p \in \mathbb{P}$, and so Proposition 1.8 follows in view of the definitions of $\nu(M, g)$ and $\sigma\left(\pi_{1}(M)\right)$.

2.4. Proof of Proposition 1.11. Consider a closed connected Riemannian manifold $(M, g)$. Let $K$ be a finite simply connected CW complex and $f: K \rightarrow M$ a continuous map, and fix $p \in \mathbb{P}$. According to (the proof of) Lemma 2.1 in [58], there exists a natural number $C_{G}$ depending only on $K$, $f, M, g$ such that given any homology class $[\psi] \in \mathrm{H}_{k}\left(\Omega(K) ; \mathbb{F}_{p}\right)$, the class $\Omega(f)_{k}([\psi])=[f \circ \psi] \in \mathrm{H}_{k}\left(\Omega(M) ; \mathbb{F}_{p}\right)$ can be represented by a $k$-cycle in $\mathcal{L}^{\left(C_{G}-2\right) k}\left(q_{0}\right) \subset \Omega^{1}\left(M, q_{0}\right)$. As in the proof of Lemma 2.13 , we then see that $[f \circ \psi]$ can be represented by a $k$-cycle $\psi^{1} \subset \mathcal{E}^{(1 / 2)\left(C_{G} k\right)^{2}}\left(q_{0}\right)$. For $h \in \pi_{1}(M)$, we denote by $\mathcal{C}_{h}$ the component of $\Omega^{1}\left(M, q_{0}\right)$ containing $h$ and abbreviate $\mathcal{E}_{h}^{a}\left(q_{0}\right)=\mathcal{E}^{a}\left(q_{0}\right) \cap \mathcal{C}_{h}$. Since $K$ is simply connected, $\psi^{1} \subset \mathcal{E}_{1}^{(1 / 2)\left(C_{G} k\right)^{2}}\left(q_{0}\right)$. We conclude that

$$
\begin{aligned}
r_{m}\left(M, K, f ; \mathbb{F}_{p}\right) & =\sum_{k=0}^{m} \operatorname{rank} \mathrm{H}_{k}\left(\Omega(f) ; \mathbb{F}_{p}\right) \\
& =\sum_{k=0}^{m} \operatorname{dim}\left(\mathrm{H}_{k}\left(\Omega(f) ; \mathbb{F}_{p}\right)\left(\mathrm{H}_{k}\left(\Omega(K) ; \mathbb{F}_{p}\right)\right)\right) \\
& \leq \sum_{k=0}^{m} \operatorname{dim} \iota_{k} \mathrm{H}_{k}\left(\mathcal{E}_{1}^{(1 / 2)\left(C_{G} m\right)^{2}}\left(q_{0}\right) ; \mathbb{F}_{p}\right)
\end{aligned}
$$

for all $m \in \mathbb{N}$. For $h \in \pi_{1}(M)$, let $\ell_{h}$ be the length of the shortest curve in $\Omega^{1}\left(M, q_{0}\right)$ representing $h$, and let $e_{h}=\frac{1}{2} \ell_{h}^{2}$ be its energy. Arguing as in the proof of Lemma 2.12 with $\epsilon=1 / 2$, we find that

$$
\operatorname{dim} \iota_{k} \mathrm{H}_{k}\left(\mathcal{E}_{1}^{a}\left(q_{0}\right) ; \mathbb{F}_{p}\right) \leq \operatorname{dim} \iota_{k} \mathrm{H}_{k}\left(\mathcal{E}_{h}^{2 a+2 e_{h}}\left(q_{0}\right) ; \mathbb{F}_{p}\right)
$$

for all $a>0$. Using this estimate and the notation from the proof of Proposition 1.8 , we can estimate

$$
\begin{aligned}
\operatorname{dim} \iota_{k} \mathrm{H}_{k}\left(\mathcal{E}^{4 a}\left(q_{0}\right) ; \mathbb{F}_{p}\right) & =\sum_{h \in \pi_{1}(M)} \operatorname{dim} \iota_{k} \mathrm{H}_{k}\left(\mathcal{E}_{h}^{4 a}\left(q_{0}\right) ; \mathbb{F}_{p}\right) \\
& \geq \sum_{h \in \pi_{1}(M)} \operatorname{dim} \iota_{k} \mathrm{H}_{k}\left(\mathcal{E}_{1}^{2 a-e_{h}}\left(q_{0}\right) ; \mathbb{F}_{p}\right) \\
& \geq \sum_{h \in \Pi_{\mathcal{E}}^{a}\left(q_{0}\right)} \operatorname{dim} \iota_{k} \mathrm{H}_{k}\left(\mathcal{E}_{1}^{a}\left(q_{0}\right) ; \mathbb{F}_{p}\right) .
\end{aligned}
$$


Together with $\# \Pi_{\mathcal{E}}^{(1 / 2)\left(C_{G} m\right)^{2}}\left(q_{0}\right)=\# \Pi_{\mathcal{L}}^{C_{G} m}\left(q_{0}\right) \geq \gamma_{S}\left(\left\lfloor C_{G} m / \ell(S, g)\right\rfloor\right)$ and (2.12), we conclude that

$$
\begin{aligned}
& \sum_{k \geq 0} \operatorname{dim} \iota_{k} \mathrm{H}_{k}\left(\mathcal{E}^{2\left(C_{G} m\right)^{2}}\left(q_{0}\right) ; \mathbb{F}_{p}\right) \\
& \quad \geq \gamma_{S}\left(\left\lfloor\frac{C_{G} m}{\ell(S, g)}\right\rfloor\right) \sum_{k \geq 0} \operatorname{dim} \iota_{k} \mathrm{H}_{k}\left(\mathcal{E}_{1}^{(1 / 2)\left(C_{G} m\right)^{2}}\left(q_{0}\right) ; \mathbb{F}_{p}\right) \\
& \quad \geq \gamma_{S}\left(\left\lfloor\frac{C_{G} m}{\ell(S, g)}\right\rfloor\right) \cdot r_{m}\left(M, K, f ; \mathbb{F}_{p}\right),
\end{aligned}
$$

and so (i) and the first inequality in (ii) of Proposition 1.11 follow in view of definitions. The second inequality in (ii) has already been obtained in Propositions 1.4 and 1.8 .

2.5. Proof of Corollary 1.14. For $M=S^{1}$, the claim follows from an elementary topological argument, see $[\mathbf{3 0}]$. We can therefore assume that $(M, g)$ is a $P$-manifold of dimension $d \geq 2$. Let $\operatorname{Ham}^{c}\left(T^{*} M\right)$ be the group of diffeomorphisms of $T^{*} M$ generated by compactly supported Hamiltonians $H:[0,1] \times T^{*} M \rightarrow \mathbb{R}$, and let $\operatorname{Symp}_{0}^{c}\left(T^{*} M\right)$ be the group of diffeomorphisms of $T^{*} M$ which are isotopic to the identity through a family of symplectomorphisms supported in a compact subset of $T^{*} M$. Since $d \geq 2$,

$$
\operatorname{Ham}^{c}\left(T^{*} M\right)=\operatorname{Symp}_{0}^{c}\left(T^{*} M\right)
$$

(see [30, Lemma 2.18]). Consider now a compactly supported symplectomorphism $\psi \in \operatorname{Symp}^{c}\left(T^{*} M\right)$ such that $[\psi]=\left[\vartheta^{m}\right] \in \pi_{0}\left(\operatorname{Symp}^{c}\left(T^{*} M\right)\right)$ for a twist $\vartheta=\vartheta_{f}$ on $T^{*} M$ and some $m \in \mathbb{Z} \backslash\{0\}$. Then, $\psi \vartheta^{-m} \in \operatorname{Symp}_{0}^{c}\left(T^{*} M\right)=$ $\operatorname{Ham}^{c}\left(T^{*} M\right)$, so that we find a compactly supported Hamiltonian function $H:[0,1] \times T^{*} M \rightarrow \mathbb{R}$ with $\varphi_{H}=\vartheta^{-m} \psi$. Then, $\psi=\vartheta^{m} \varphi_{H}=\varphi_{m f} \varphi_{H}=\varphi_{K}$, where

$$
K(t, q, p)=m f(|p|)+H\left(t, \vartheta_{f}^{-m}(q, p)\right) .
$$

Choose $r_{k} \geq 1$ so large that $H$ is supported in $[0,1] \times\left\{|p| \leq r_{K}\right\}$. Since $\vartheta_{f}^{-m}$ preserves the levels $\{|p|=$ const $\}$, we then have $K(t, q, p)=m f(|p|)$ for $|p| \geq r_{k}$. Since $f^{\prime}\left(r_{K}\right)=1$ and $m \neq 0$, Theorem 1.2 (ii) applies and yields $\check{s}_{\text {fibre }}\left(\varphi_{K} ; r_{K}\right) \geq 1$, as claimed.

\section{More on $P$-manifolds}

Much information on $P$-manifolds can be found in the book [8] and in Section 10.10 of $[\mathbf{7}]$. In this section, we give a few additional results on the topology of $P$-manifolds. It is easy to see that $P$-manifolds of dimension at least 2 have finite fundamental group [8, 7.38].

Proposition 3.1. Let $(M, g)$ be a P-manifold of dimension at least 2. Then, $M$ is elliptic, and $c(M)=\rho(M)=1$. 
Proof. Recall from Discussion 1.15 (a) in Section 1.7 that an elementary computation shows $\check{s}_{\text {fibre }}\left(\vartheta_{f}\right)=1$ for every twist on $T^{*} M$. The proposition thus follows from Discussion 1.5 (ii).

The above proof of the inequality $\rho(M) \leq 1$ relies on Floer homology and Gromov's theorem invoked earlier. We now prove this inequality by elementary means. A version of the argument proves $c(M) \leq 1$, cf. [66, p. 129].

An elementary proof of $\rho(M) \leq 1$. Recall that we scaled $g$ such that all unit-speed geodesics have minimal period 1 . For such a geodesic $\gamma: \mathbb{R} \rightarrow M$ and $t>0$, we let ind $\gamma(t)$ be the number of linearly independent Jacobi fields along $\gamma(s), s \in[0, t]$, which vanish at $\gamma(0)$ and $\gamma(t)$. If ind $\gamma(t)>0$, then $\gamma(t)$ is said to be conjugate to $\gamma(0)$ along $\gamma$. The index of $\left.\gamma\right|_{[0, a]}$ defined as

$$
\text { ind }\left.\gamma\right|_{[0, a]}=\sum_{t \in] 0, a[} \text { ind } \gamma(t)
$$

is a finite number, and according to [8, 1.98 and 7.25], the number $k=$ ind $\left.\gamma\right|_{[0,1]}$ is the same for all unit-speed geodesics $\gamma$ on $(M, g)$. Fix now $q_{0} \in M$ and choose $q_{1}$ which is not conjugate to $q_{0}$. Then, there are only finitely many geodesic segments $\gamma_{1}, \ldots, \gamma_{n}$ from $q_{0}$ to $q_{1}$ of length smaller than 1 , see $[8,7.41]$. Let $k_{1}, \ldots, k_{n}$ be their indices. The energy functional on $\Omega^{1}\left(M ; q_{0}, q_{1}\right)$ is Morse with indices $k_{i}+l(d-1+k)$, where $1 \leq i \leq n$ and $l=$ $0,1,2, \ldots$. Since $d-1+k \geq 1$, we conclude that $\operatorname{dim} \mathrm{H}_{j}\left(\Omega^{1}\left(M ; q_{0}, q_{1}\right) ; \mathbb{F}_{p}\right) \leq$ $n$ for all $j \in \mathbb{N}$ and $p \in \mathbb{P}$. Recall now that the inclusion $\Omega^{1}\left(M ; q_{0}, q_{1}\right) \hookrightarrow$ $\Omega\left(M ; q_{0}, q_{1}\right)$ is a homotopy equivalence. Therefore, $\operatorname{dim}_{j}\left(\Omega(M) ; \mathbb{F}_{p}\right) \leq n$ for all $j$ and $p$, and so $\rho(M) \leq 1$, as claimed.

The subsequent results on the topology of $P$-manifolds will rely only on the possibly weaker assumption $\rho(M)=1$. We shall therefore assume throughout that $M$ is a closed connected manifold with finite $\pi_{1}(M)$ and $\rho(M)=1$. We first consider the case that $M$ is simply connected. All such known $P$-manifolds are diffeomorphic to a CROSS $S^{d}, \mathbb{C P}^{n}, \mathbb{H} \mathrm{P}^{n}$, or $\mathbb{C}_{a} \mathrm{P}^{2}$ with its standard smooth structure.

Proposition 3.2 [49]. Assume that $M$ is simply connected. Then $\rho(M)=1$ if and only if $M$ has the integral cohomology ring of a CROSS.

Corollary 3.3. A simply connected manifold with $\rho(M)=1$ is either homeomorphic to $S^{d}$ or homotopy equivalent to $\mathbb{C P}^{n}$ or has the integral cohomology ring of $\mathbb{H}^{n}$ or $\mathbb{C} a \mathrm{P}^{2}$.

Proof. If $\mathrm{H}^{*}(M ; \mathbb{Z})=\mathrm{H}^{*}\left(S^{d} ; \mathbb{Z}\right)$, then $M$ is a simply connected homotopy sphere by Hurewicz's theorem and hence homeomorphic to $S^{d}$ by the proof of the Poincaré conjecture. If $\mathrm{H}^{*}(M ; \mathbb{Z})=\mathrm{H}^{*}\left(\mathbb{C P}^{n} ; \mathbb{Z}\right)$, then $M$ is homotopy equivalent to $\mathbb{C P}^{n}$ by a theorem of Klingenberg [42]. 
In small dimensions, we obtain

Corollary 3.4. Assume that $M$ is a simply connected d-manifold with $\rho(M)=1$.

If $d=3$, then $M$ is diffeomorphic to $S^{3}$.

If $d=4$, then $M$ is homeomorphic to $S^{4}$ or $\mathbb{C P}^{2}$.

If $d=5$, then $M$ is diffeomorphic to $S^{5}$.

If $d=6$, then $M$ is diffeomorphic to $S^{6}$ or homotopy equivalent to $\mathbb{C P}^{3}$.

If $d=7$, then $M$ is homeomorphic to $S^{7}$.

\section{Remark 3.5.}

(a) According to [73], the set of closed smooth 6-manifolds homotopy equivalent to $\mathbb{C P}^{3}$ splits into infinitely many diffeomorphism types $M_{k}$, $k \in \mathbb{Z}$; they are determined by their first Ponrjagin class $p_{1}\left(M_{k}\right)=$ $(48 k+4) a^{2}$, where $a$ is a generator of $\mathrm{H}^{2}\left(M_{k} ; \mathbb{Z}\right)$. It would be interesting to see whether $\mathbb{C P}^{3}=M_{0}$ is the only diffeomorphism type carrying a $P$-metric.

(b) Corollaries 3.3 and 3.4 partially answer Question 1.16 (a) in the simply connected case. There are, however, closed connected 8- and 16manifolds $M$ with the integral cohomology ring of $\mathbb{H}^{2}$ and $\mathbb{C} a \mathrm{P}^{2}$ (and hence with $\rho(M)=1$ ) which are not homotopy equivalent to $\mathbb{H}^{2}$ and $\mathbb{C} a \mathrm{P}^{2}$, see $[\mathbf{6}, \mathbf{1 7}]$, and it is unknown whether these homotopy types can be realized by $P$-manifolds.

In order to study manifolds with $\rho(M)=1$ and non-trivial $\pi_{1}(M)$, we shall need the following well-known result, whose proof is given for the reader's convenience.

Proposition 3.6. Assume that $X$ is a retract of a finite simplicial complex whose integral cohomology ring is the one of $\mathbb{C P}^{2 n}$ with $n \geq 1$ or of $\mathbb{H} \mathrm{P}^{n}$ with $n \geq 2$ or of $\mathbb{C}_{a} \mathrm{P}^{2}$. Then each continuous self mapping of $X$ has a fixed point.

Proof. We closely follow [37]. The cohomology $\operatorname{ring} \mathrm{H}^{*}(X ; \mathbb{Z}) \cong\langle\alpha\rangle /\left\langle\alpha^{n+1}\right\rangle$ is generated by an element $\alpha \in \mathrm{H}^{k}(X ; \mathbb{Z})$, where $k \in\{2,4,8\}$. If $\mathrm{H}^{*}(X ; \mathbb{Z})$ is the cohomology ring of $\mathbb{C P}^{2 n}$ with $n \geq 1$ or of $\mathbb{H} \mathrm{P}^{n}$ with $n$ even or of $\mathbb{C} a \mathrm{P}^{2}$, the claim thus follows at once from the Lefschetz fixed point theorem, see, for instance, $[37$, Theorem 2.C.3]. Assume now that $X$ has the integral cohomology ring of $\mathbb{H} \mathrm{P}^{n}, n \geq 3$. The image of $\alpha$ in $\mathrm{H}^{4}\left(X ; \mathbb{Z}_{3}\right)$, which is again denoted by $\alpha$, generates $\mathrm{H}^{*}\left(X ; \mathbb{Z}_{3}\right)$. Let $f$ be a continuous self mapping of $X$, and define its degree $d_{f}=d_{3}(f) \in \mathbb{Z}_{3}$ by $f^{*} \alpha=d_{f} \alpha$. The following lemma shows that $d_{f}=0$ or $d_{f}=1$. If $d_{f}=0$, the Lefschetz number of $f^{*}: \mathrm{H}^{*}(X ; \mathbb{Z}) \rightarrow \mathrm{H}^{*}(X ; \mathbb{Z})$ is $\sum_{j=0}^{n}(3 l)^{j}$ for some $l \in \mathbb{Z}$, and if $d_{f}=1$, it is $\sum_{j=0}^{n}(1+3 l)^{j}$ for some $l \in \mathbb{Z}$. Since both numbers are not 0 , the proposition again follows from the Lefschetz fixed point theorem. 
Lemma 3.7. The degree $d_{f}$ satisfies $d_{f}=d_{f}^{2}$ in $\mathbb{Z}_{3}$.

Proof. Recall that the Steenrod power operations for the odd prime 3 are maps $P^{i}: \mathrm{H}^{n}\left(X ; \mathbb{Z}_{3}\right) \rightarrow \mathrm{H}^{n+4 i}\left(X ; \mathbb{Z}_{3}\right)$ satisfying various axioms, see $[\mathbf{3 7}$, Section 4.L]. Define $x \in \mathbb{Z}_{3}$ by

$$
P^{1}(\alpha)=x \alpha^{2} .
$$

From naturality $P^{1} f^{*} \alpha=f^{*} P^{1} \alpha$, we obtain $d_{f} x \alpha^{2}=x d_{f}^{2} \alpha^{2}$. Since $n \geq 2$, we have $\alpha^{2} \neq 0$, so that

$$
x\left(d_{f}-d_{f}^{2}\right)=0 \quad \text { in } \mathbb{Z}_{3} .
$$

It remains to show that $x \neq 0$ in $\mathbb{Z}_{3}$. Using the Cartan formula for $P^{1}$, we compute $P^{1} P^{1} \alpha=2 x^{2} \alpha^{3}$, and since $\alpha$ has degree 4 , we have $P^{2} \alpha=\alpha^{3}$. The Adem relation $P^{1} P^{1}=-P^{2}$ applied to $\alpha$ thus yields $2 x^{2} \alpha^{3}=-\alpha^{3}$. Since $n \geq 3$, we have $\alpha^{3} \neq 0$, so that $x \neq 0$. This proves the lemma.

Every compact, locally contractible space that embeds into some $\mathbb{R}^{n}$, and hence every closed manifold, is a retract of a finite simplicial complex, see [37, Theorem A.7].

Consider now a manifold with $\rho(M)=1$ and non-trivial $\pi_{1}(M)$. The only known such Riemannian $P$-manifolds are the spherical space forms $S^{2 n+1} / \Gamma$ classified in [75], the standard $\mathbb{R P}^{2 n}=S^{2 n} / \mathbb{Z}_{2}$, and the quotient $\mathbb{C P} P^{2 n+1} / \mathbb{Z}_{2}$ obtained from the involution

$$
\left[z_{0}: z_{1}: \cdots: z_{2 n+1}\right] \longmapsto\left[\bar{z}_{1}:-\bar{z}_{0}: \bar{z}_{3}:-\bar{z}_{2}: \cdots: \bar{z}_{2 n+1}:-\bar{z}_{2 n}\right] .
$$

The following corollary gives a partial answer to Question 1.16(a) in the non-simply connected case.

Corollary 3.8. Assume that $M$ is a d-manifold with $\rho(M)=1$ and nontrivial finite $\pi_{1}(M)$, and let $\widetilde{M}$ be its universal cover.

(a) If $d$ is odd, then $\widetilde{M}$ is homeomorphic to $S^{d}$. If, in addition, $\pi_{1}(M)$ is cyclic, then $M$ is homotopy equivalent to a lens space.

(b) If $d$ is even, then either $\widetilde{M}$ is homeomorphic to $S^{d}$ and $M$ is homotopy equivalent to $\mathbb{R P}^{d}$, or $\widetilde{M}$ is homotopy equivalent to $\mathbb{C P}^{2 n+1}$ and $\pi_{1}(M)=\mathbb{Z}_{2}$.

Proof. According to Lemma 2.12, we also have $\rho(\widetilde{M})=1$. Since $\pi_{1}(M)$ acts smoothly on $\widetilde{M}$ without fixed points, Corollary 3.3 and Proposition 3.6 show that $\widetilde{M}$ is homeomorphic to $S^{d}$ or homotopy equivalent to $\mathbb{C P}^{2 n+1}$.

Assume now that $d$ is odd and that $\pi_{1}(M)=\mathbb{Z}_{q}$ is cyclic. According to $[\mathbf{1 1}$, Lemma 1], the quotient of a topological $d$-sphere by a smooth fixed point free action of $\mathbb{Z}_{q}$ is homotopy equivalent to a lens space.

Assume finally that $d$ is even. According to [46, IV.3.1.], the quotient of a topological $d$-sphere by a smooth fixed point free action of $\mathbb{Z}_{2}$ is homotopy 
equivalent to $\mathbb{R P}^{d}$. It thus remains to show that $\pi_{1}(M)=\mathbb{Z}_{2}$. Denote the diffeomorphism of $\widetilde{M}$ induced by $\varphi \in \pi_{1}(M)$ also by $\varphi$. If $\varphi$ is orientation preserving, again the Lefschetz fixed point theorem implies that $\varphi$ has a fixed point, so that $\varphi$ is the identity. Therefore, each $\varphi \in \pi_{1}(M) \backslash\{\operatorname{id}\}$ is orientation reversing and of order 2 . This implies at once that there is only one $\varphi \in \pi_{1}(M) \backslash\{$ id $\}$.

\section{Remark 3.9.}

(a) The classification problem of homotopy lens spaces of dimension $\geq 5$ and with fundamental group $\mathbb{Z}_{q}, q$ odd, has been studied in [12] using surgery theory. For the classification of lens spaces, we refer to $[\mathbf{5 2}, \mathbf{7 0}]$.

(b) ad (b). Exotic spheres of even dimension $2 n \in\{6, \ldots, 14\}$ admit no fixed point free involution, see [46, V.6.2.]. It is conceivable that this is true in all even dimensions. There are smooth free involutions of $S^{4}$ whose quotient is homotopy equivalent but not diffeomorphic to $\mathbb{R} \mathrm{P}^{4}$, see $[\mathbf{1 3}, \mathbf{2 1}]$, and for $k \geq 2$, there are smooth free involutions of homotopy $S^{4 k}$ 's whose quotient is homotopy equivalent but not diffeomorphic to $\mathbb{R P}^{4 k}$, see $[\mathbf{2 2}]$. All homotopy $\mathbb{C P}^{3}$ 's admit smooth free involutions $[\mathbf{5 9}, \mathbf{6 1}]$.

(c) Assume that in the situation of Corollary 3.8, there exists a point $p \in M$ such that all geodesics through $p$ are closed and have equal length. Then $M$ is diffeomorphic to $\mathbb{R P}^{d}$, see [8, 7.23].

\section{Outlook}

The conceptual point of view of this paper was to look at entropy-type quantities which are well understood for geodesic flows and to establish the lower bounds for these quantities known for geodesic flows for arbitrary classical Hamiltonian systems by interpreting these quantities in Floer homological terms and by using the deformation invariance of Floer homology. We were able to do this only for Hamiltonians meeting (1.4) by using the Convexity Lemma 2.2. Already the autonomous Hamiltonians $H(q, p)=\frac{1}{2}|p-A(q)|^{2}+V(q)$ modelling the dynamics of a particle in a magnetic potential $A(q)$ and a scalar potential $V(q)$ do not meet (1.4), and the Convexity Lemma fails for such Hamiltonians. Lower bounds for the topological entropy of Reeb flows on fiberwise star-shaped hypersurfaces in $T^{*} M$ will be obtained in $[\mathbf{4 7}]$ by bringing into play the action filtration on Floer homology and the sandwiching method.

\section{Acknowledgements}

We wish to thank the referee of our paper [30] for suggesting the present approach to Corollary 1.14. Another important stimulus was Gabriel Paternain's beautiful lecture on topological entropy at Max Planck Institute 
Leipzig in November 2004. We warmly thank Gabriel for corrections and many remarks, which considerably improved a first draft of the paper. Most of this paper was written at the turn of the year 2004/2005 at Hokkaido University at Sapporo. We cordially thank Kaoru Ono for valuable discussions and for his generous hospitality and Theo Bühler, Steve Halperin, Otto van Koert, Leonardo Macarini, and Matthias Schwarz for their help. This work was supported by the Japanese Society for Promotion of Science (UF) and the Deutsche Forschungsgemeinschaft (FS).

\section{References}

[1] A. Abbondandolo and M. Schwarz, On the Floer homology of cotangent bundles, Comm. Pure Appl. Math. 59 (2006), 254-316.

[2] J. Anderson and G. Paternain, The minimal entropy problem for 3-manifolds with zero simplicial volume, Geometric methods in dynamics. I, Astérisque 286 (2003), xvii-xviii, 63-79.

[3] V.I. Arnol'd, Some remarks on symplectic monodromy of Milnor fibrations, The Floer memorial volume, Progr. Math. 133, Birkhäuser, Basel, 1995, 99-103.

[4] H. Bass, The degree of polynomial growth of finitely generated nilpotent groups, Proc. Lond. Math. Soc. 25 (1972), 603-614.

[5] V. Benci, Periodic solutions of Lagrangian systems on a compact manifold, J. Diff. Eq. 63 (1986), 135-161.

[6] L. Bérard-Bergery, Quelques exemples de variétés riemanniennes où toutes les géodésiques issues d'un point sont fermées et de même longueur, suivis de quelques résultats sur leur topologie, Ann. Inst. Fourier 27 (1977), 231-249.

[7] M. Berger, A panoramic view of Riemannian geometry, Springer-Verlag, Berlin, 2003.

[8] A. Besse, Manifolds all of whose geodesics are closed, Ergebnisse der Mathematik und ihrer Grenzgebiete 93, Springer, Berlin-New York, 1978.

[9] P. Biran, L. Polterovich and D. Salamon, Propagation in Hamiltonian dynamics and relative symplectic homology, Duke Math. J. 119 (2003), 65-118.

[10] F. Bonahon, Geometric structures on 3-manifolds, Handbook of Geometric Topology, North-Holland, Amsterdam, 2002, 93-164.

[11] W. Browder, Free $Z_{p}$-actions on homotopy spheres, in Topology of Manifolds (Proc. Inst., Univ. of Georgia, Athens, GA, 1969) Markham, Chicago, Ill, 1970, 217-226.

[12] W. Browder, T. Petrie and C.T.C. Wall, The classification of free actions of cyclic groups of odd order on homotopy spheres, Bull. Amer. Math. Soc. 77 (1971), $455-459$

[13] S. Cappell and J. Shaneson, Some new four-manifolds, Ann. Math. 104 (1976), 61-72.

[14] K. Cieliebak, A. Floer and H. Hofer, Symplectic homology. II. A general construction, Math. Z. 218 (1995), 103-122.

[15] E.I. Dinaburg, A connection between various entropy characterizations of dynamical systems, Izv. Akad. Nauk SSSR Ser. Mat. 35 (1971), 324-366.

[16] P. de la Harpe, Topics in geometric group theory, Chicago Lectures in Mathematics, University of Chicago Press, Chicago, IL, 2000. 
[17] J. Eells and N. Kuiper, Manifolds which are like projective planes, Inst. Hautes Études Sci. Publ. Math. 14 (1962), 5-46.

[18] Y. Félix, S. Halperin and J.-C. Thomas, Elliptic spaces, Bull. Amer. Math. Soc. 25 (1991), 69-73.

[19] Y. Félix, S. Halperin and J.-C. Thomas, Elliptic spaces. II, Enseign. Math. 39 (1993), 25-32.

[20] Y. Félix, S. Halperin and J.-C. Thomas, Rational homotopy theory, Graduate Texts in Mathematics 205, Springer-Verlag, New York, 2001.

[21] R. Fintushel and R. Stern, An exotic free involution on $S^{4}$, Ann. Math. 113 (1981), 357-365.

[22] R. Fintushel and R. Stern, Smooth free involutions on homotopy $4 k$-spheres, Michigan Math. J. 30 (1983), 37-51.

[23] A. Floer, A relative Morse index for the symplectic action, Comm. Pure Appl. Math. 41 (1988), 393-407.

[24] A. Floer, The unregularized gradient flow of the symplectic action, Comm. Pure Appl. Math. 41 (1988), 775-813.

[25] A. Floer, Morse theory for Lagrangian intersections, J. Diff. Geom. 28 (1988), 513-547.

[26] A. Floer, Witten's complex and infinite-dimensional Morse theory, J. Diff. Geom. 30 (1989), 207-221.

[27] A. Floer, H. Hofer and D. Salamon, Transversality in elliptic Morse theory for the symplectic action, Duke Math. J. 80 (1995), 251-292.

[28] U. Frauenfelder and F. Schlenk, Hamiltonian dynamics on convex symplectic manifolds, Israel J. Math. (2006), to appear, math.SG/0303282.

[29] U. Frauenfelder and F. Schlenk, Slow entropy and symplectomorphisms of cotangent bundles, math.SG/0404017.

[30] U. Frauenfelder and F. Schlenk, Volume growth in the component of the Dehn-Seidel twist, Geom. Funct. Anal. 15 (2005), 809-838.

[31] U. Frauenfelder and F. Schlenk, Gaps for homological growth and volume growth, in preparation.

[32] R. Grigorchuk, On growth in group theory (Proc. ICM, I, Kyoto, 1990), Math. Soc. Japan, Tokyo, 1991, 325-338.

[33] M. Gromov, Homotopical effects of dilatation, J. Diff. Geom. 13 (1978), 303-310.

[34] M. Gromov, Groups of polynomial growth and expanding maps, Inst. Hautes Études Sci. Publ. Math. 53 (1981), 53-73.

[35] M. Gromov, Metric structures for Riemannian and non-Riemannian spaces, Progr. Math. 152, Birkhäuser, Basel, 1999.

[36] Y. Guivarc'h, Groupes de Lie à croissance polynomiale, C. R. Acad. Sci. Paris Sér. A-B 271 (1970), 237-239; 272 (1971), 1695-1696.

[37] A. Hatcher, Algebraic topology, Cambridge University Press, Cambridge, 2002.

[38] J.A. Hillman, A remark on 4-manifolds with zero entropy metrics, University of Sydney, Preprint, 2005.

[39] A. Katok and B. Hasselblatt, Introduction to the modern theory of dynamical systems, Encyclopedia of Mathematics and its Applications 54, Cambridge University Press, Cambridge, 1995. 
[40] A. Katok and J.P. Thouvenot, Slow entropy type invariants and smooth realization of commuting measure-preserving transformations, Ann. Inst. H. Poincaré Probab. Statist. 33 (1997), 323-338.

[41] M. Khovanov and P. Seidel, Quivers, Floer cohomology, and braid group actions, J. Amer. Math. Soc. 15 (2002), 203-271.

[42] W. Klingenberg, Manifolds with restricted conjugate locus, Ann. Math. 78 (1963), $527-547$.

[43] W. Klingenberg, Lectures on closed geodesics, Grundlehren der Mathematischen Wissenschaften 230, Springer-Verlag, Berlin-New York, 1978.

[44] W. Klingenberg, Riemannian geometry, de Gruyter Studies in Mathematics, 1, 2nd ed., de Gruyter, Berlin, 1995.

[45] P. Lambrechts, The Betti numbers of the free loop space of a connected sum, J. Lond. Math. Soc. 64 (2001), 205-228.

[46] S. López de Medrano, Involutions on manifolds, Ergebnisse der Mathematik und ihrer Grenzgebiete 59, Springer-Verlag, New York-Heidelberg, 1971.

[47] L. Macarini and F. Schlenk, Positive topological entropy of Reeb flows on fiberwise starshaped energy surfaces, in preparation.

[48] E. Mazet, Sur les travaux de Milnor-Wolf. Variétés à courbure négative, Papers from the Seminar on Riemannian Geometry, 1970/1971, 2nd ed., Publications Mathématiques de l'Université Paris VII, 8, Université de Paris VII, U.E.R. de Mathématiques, Paris, 1979.

[49] J. McCleary, On the mod p Betti numbers of loop spaces, Invent. Math. 87 (1987), 643-654.

[50] C. McGibbon and C. Wilkerson, Loop spaces of finite complexes at large primes, Proc. Amer. Math. Soc. 96 (1986), 698-702.

[51] J. Milnor, Morse theory, Annals of Mathematics Studies 51, Princeton University Press, Princeton, NJ, 1963.

[52] J. Milnor, Whitehead torsion, Bull. Amer. Math. Soc. 72 (1966), 358-426.

[53] J. Milnor, A note on curvature and fundamental group, J. Diff. Geom. 2 (1968), $1-7$.

[54] S. Newhouse, Entropy and volume, Ergodic Theory Dynam. Systems 8 $^{*}$ (1988), Charles Conley Memorial Issue, 283-299.

[55] G. Paternain, Topological entropy for geodesic flows on fibre bundles over rationally hyperbolic manifolds, Proc. Amer. Math. Soc. 125 (1997), 2759-2765.

[56] G. Paternain, Geodesic flows, Progress in Mathematics 180, Birkhäuser Boston, Inc., Boston, MA, 1999.

[57] G. Paternain and J. Petean, Minimal entropy and collapsing with curvature bounded from below, Invent. Math. 151 (2003), 415-450.

[58] G. Paternain and J. Petean, Zero entropy and bounded topology, Comment. Math. Helv. 81 (2006), 287-304.

[59] T. Petrie, Involutions on homotopy complex projective spaces and related topics (Proc. Second Conference on Compact Transformation Groups) Lecture Notes in Math. 298, Springer, Berlin, 1972, pp. 234-259.

[60] L. Polterovich, Growth of maps, distortion in groups and symplectic geometry, Invent. Math. 150 (2002), 655-686. 
[61] R. Sady, Free involutions of complex projective spaces, Michigan Math. J. 24 (1977), 51-64.

[62] D. Salamon, Morse theory, the Conley index and Floer homology, Bull. Lond. Math. Soc. 22 (1990), 113-140.

[63] D. Salamon and J. Weber, Floer homology and the heat flow, Geom. Funct. Anal., to appear, math.SG/0304383.

[64] M. Schwarz, Morse homology, Progress in Mathematics 111, Birkhäuser Verlag, Basel, 1993.

[65] P. Seidel, Lagrangian two-spheres can be symplectically knotted, J. Diff. Geom. 52 (1999), 145-171.

[66] P. Seidel, Graded Lagrangian submanifolds, Bull. Soc. Math. France 128 (2000), 103-149.

[67] J.-P. Serre, Homologie singulière des espaces fibrés. Applications, Ann. Math. 54 (1951), 425-505.

[68] S. Smale, On the structure of 5-manifolds, Ann. Math. 75 (1962), 38-46.

[69] J. Tits, Appendix to: "Groups of polynomial growth and expanding maps" [Inst. Hautes Études Sci. Publ. Math. 53 (1981), 53-73] by M. Gromov, Inst. Hautes Études Sci. Publ. Math. 53 (1981), 74-78.

[70] V. Turaev, Introduction to combinatorial torsions. Lectures in Mathematics ETH Zürich, Birkhäuser Verlag, Basel, 2001.

[71] C. Viterbo, Generating Functions in Symplectic Topology and Applications (Proc. ICM 94, 1, Zürich), Birkhäuser, Basel, 1995, 537-547.

[72] C. Viterbo, Functors and computations in Floer homology with applications, I, Geom. Funct. Anal. 9 (1999), 985-1033.

[73] C.T.C. Wall, Classification problems in differential topology. V. On certain 6manifolds, Invent. Math. 1 (1966), 355-374.

[74] J. Wolf, Growth of finitely generated solvable groups and curvature of Riemannian manifolds, J. Diff. Geom. 2 (1968), 421-446.

[75] J. Wolf, Spaces of Constant Curvature. Fifth edition. Publish or Perish, Inc., Houston, 1984.

[76] Y. Yomdin, Volume growth and entropy, Israel J. Math. 57 (1987), 285-300.

Mathematisches Institut Der Universität München

THERESIENSTR. 39

80333 München, Germany

E-mail address: urs.frauenfelder@mathematik.uni-muenchen.de

DÉPARTEMENT DE MAThÉMATiqUeS

Université LiBre de BRuXelles

CP 218

Boulevard Du TRIOMPhe

1050 Bruxelles, Belgium

E-mail address: fschlenk@ulb.ac.be

Received 15 December 2005 